\title{
Finite-Horizon $H_{\infty}$ State Estimation for Stochastic Coupled Networks with Random Inner Couplings using Round-Robin Protocol
}

\author{
Yun Chen, Zidong Wang, Licheng Wang and Weiguo Sheng
}

\begin{abstract}
This paper is concerned with the problem of finitehorizon $H_{\infty}$ state estimation for time-varying coupled stochastic networks through the Round-Robin scheduling protocol. The inner coupling strengths of the considered coupled networks are governed by a random sequence with known expectations and variances. For the sake of mitigating the occurrence probability of network-induced phenomena, the communication network is equipped with the Round-Robin protocol that schedules the signal transmissions of the sensors' measurement outputs. By using some dedicated approximation techniques, an uncertain auxiliary system with stochastic parameters is established where the multiplicative noises enter into the coefficient matrix of the augmented disturbances. With the established auxiliary system, the desired finite-horizon $H_{\infty}$ state estimator is acquired by solving coupled backward Riccati equations, and the corresponding recursive estimator design algorithm is presented that is suitable for online application. The effectiveness of the proposed estimator design method is validated via a numerical example.
\end{abstract}

Index Terms-Stochastic coupled networks; finite-horizon $H_{\infty}$ estimation; random inner couplings; Round-Robin protocol; backward Riccati difference equations.

\section{INTRODUCTION}

With the springing up of the discipline of complexity science over the past few decades, complex networks have become a research hotspot due mainly to their wide range of applications in our daily life. Generally speaking, a typical complex network consists of a large number of nodes and edges which can be used to model individual systems and their interconnections, respectively. Based on this prominent structure, many complex systems can be described by complex

This work was supported in part by the Zhejiang Provincial Natural Science Foundation of China under grant LR16F030003, in part by the National Natural Science Foundation of China under Grant 61973102, Grant 61873148, Grant 61933007, Grant 61873082, and Grant U1509205, in part by the China Postdoctoral Science Foundation under Grant 2019TQ0202, in part by the Shanghai Pujiang Program of China under Grant 19PJ1408100, in part by the Royal Society of the UK, and in part by the Alexander von Humboldt Foundation of Germany. (Corresponding author: Zidong Wang).

Y. Chen is with the Key Lab for IoT and Information Fusion Technology of Zhejiang, School of Automation, Hangzhou Dianzi University, Hangzhou 310018, China. (Email: yunchen@hdu.edu.cn)

Z. Wang is with the College of Electrical Engineering and Automation, Shandong University of Science and Technology, Qingdao 266590, China. $\mathrm{He}$ is also with the Department of Computer Science, Brunel University London, Uxbridge, Middlesex, UB8 3PH, United Kingdom. (Email: Zidong.Wangebrunel.ac.uk)

L. Wang is with Shanghai Key Lab of Modern Optical System, Department of Control Science and Engineering, University of Shanghai for Science and Technology, Shanghai 200093, China. (Email: wanglicheng1217@163.com)

W. Sheng is with the Department of Computer Science, Hangzhou Normal University, Hangzhou 311121, China. (Email: weiguouk@hotmail.com) network models with examples including nervous systems, computer networks, transportation networks and social networks. Compared with those individual systems, complex networks exhibit features such as strong couplings, inherent nonlinearities as well as large scales that contribute greatly to the complexities in the dynamical behaviors and, therefore, there appears to be an urgent demand in understanding the dynamic evolution of complex networks. In recent years, tremendous research efforts have been devoted to the dynamic analysis issues for complex networks such as stability, synchronization, state estimation and pining control, see e.g. [6], [14]-[16], [18], [22], [26], [30], [31], [45].

It is well recognized that, as an indispensable part of the coupled networks, the coupling strengths have an essential impact on both the topology connection and the dynamics of complex networks. In most of the existing literature, an implicit assumption is that coupling strengths are deterministic yet fixed. In some practical situations, however, the inner connections between nodes might be uncertain and expose certain switching/random behaviors owing to a variety of reasons such as network congestion, random failures, unknown but sudden changes of the working conditions as well as the unexpected environmental changes. In view of this, particular attention has been paid to the investigation on the impact from the uncertain/random/switching couplings on network dynamics [3], [6], [15], [19], [20], [30]. For example, in [22], the coupling strengths have been characterized as a uncertain term and the resulting uncertainties have been dealt with using the $H_{\infty}$ performance requirements on the filtering error dynamics. The stochastic coupling (either inter-coupling or outercoupling) connecting strengths has been considered in [26]. In [10], the switching coupling strengths obeying a discretetime Markov chain have been proposed, whose influences on both the variance constraint and $H_{\infty}$ performance have been analyzed.

When analyzing the dynamical performance of complex networks, it is often a prerequisite that the state information of all nodes is available especially for certain tasks such as synchronization and consensus. Unfortunately, this is not always possible due to unknown/unpredictable environmental changes or limits of measurement technologies/expenses. Therefore, the state estimation problem of complex networks has become an issue of primary importance that has recently aroused much research interest with many results reported in the literature, see e.g. [26], [31], [39], [40]. It is worth noting that most results on the state estimation of complex networks have 
been concerned with the time-invariant systems. However, in practical situations, the evolution of the networks is very likely to be time-varying with the change of the working environment. As such, the finite-horizon state estimation problem for the time-varying complex networks, whose main idea is to guarantee a satisfactory transient performance over a certain period time, has received some initial research attention [29], [33], [35]. Up to now, several effective approaches/techniques have been developed, e.g. the recursive linear matrix inequality (RLMI) approach [5], [42], the Krein-space theory [21] and the backward coupled recursive Riccati equation (BCRRE) method [26], among which the BCRRE technique has proven to be particularly efficient in facilitating the online applications for nonlinear time-varying systems [8].

With the increase of the network scale, the coupled nodes lead inevitably to a large amount of information exchange demanding a great deal of communication resources that are usually limited. In fact, the inherently limited bandwidth of the communication network is very likely to create obstacles for sustaining the ever-expedited data interactions for a large scale network. To cope with the sparsity of the communication resources, some efficient data transmission strategies have been proposed. For example, with purpose to reduce the communication frequency, the event-triggered strategy has been developed where the information is transmitted only when certain prescribed event is satisfied, see e.g. [11], [15], [27], [32], [44]. Another data scheduling strategy that has recently begun to receive some research attention is the so-called communication protocol whose main idea is to only grant the "selected" data the permission to occupy the communication channel, thereby effectively preventing the undesired data collisions [1]. In general, the commonly deployed communication protocols include the Round-Robin protocol (RRP) [25], [36], [38], [41], [43], the Try-Once-Discard protocol [17] and the random access protocol [48]. Among others, the RRP is a kind of periodic protocols under which all signals are transmitted in a given circular fashion. Due to its structural simplicity and convenient implementation, the RRP has been widely applied in industry with a surge of interest in dynamics analysis (e.g. state estimation) problems for a variety of complex networks, see e.g. [23], [26], [28].

Motivated by the above discussions, in this paper, we like to initiate a systematic investigation on the new yet challenging problem of RRP-based finite-horizon $H_{\infty}$ state estimation problem for a class of time-varying stochastic coupled networks subject to random inner coupling strengths. The main contributions of this paper can be summarized as follows. 1) The coupled networks are quite comprehensive that involves state-dependent multiplicative noises and random inner coupling connections. 2) The RRP is adopted to schedule the measurement data of the underlying stochastic complex networks. 3) An auxiliary stochastic parameter system is dedicatedly developed so as to facilitate the evaluation of the $H_{\infty}$ performance over a finite horizon. 4) A novel BCRRE approach is put forward to design the gain parameters of the desired state estimator in a recursive manner.

The remainder of this paper is organized as follows. Section II addresses the problem statement and preliminaries of this paper. The main results are given in Section III. A numerical example is provided in Section IV and the conclusion is drawn in Section V.

Notation: Throughout this paper, the notations used are standard. $\mathbb{R}^{n}$ denotes the $n$-dimensional Euclidean space. $I$ and 0 are used to indicate an identity matrix and a zero matrix with appropriate dimensions, respectively. $\|\cdot\|$ designates the Euclidean norm. $\mathbf{E}\{\cdot\}$ denotes the mathematical expectation. $\operatorname{diag}\{\cdots\}$ represents a block diagonal matrix. The Kronecker product of the two matrices $A$ and $B$ is represented as $A \otimes B$. $(A)^{\dagger}$ is the Moore-Penrose pseudo inverse of matrix $A . P^{i j}$ is the $(i, j)$-th entry of the matrix $P . l_{2}[0, T]$ refers to a square summable space over the finite time interval $[0, T]$.

\section{Problem Formulation And Preliminaries}

\section{A. The Complex Network Model}

Consider the following coupled network with $N$ nodes:

$$
x_{i, k+1}=f\left(x_{i, k}\right)+\sum_{j=1}^{N} r_{i j} G_{k} x_{j, k}+B_{i, k} v_{k}+h_{i}\left(x_{i, k}\right) w_{k}
$$

where $x_{i, k} \in \mathbb{R}^{n}(i \in \mathcal{N}=\{1,2, \cdots, N\})$ is the state vector of node $i, v_{k} \in \mathbb{R}^{n_{v}}$ is the bounded exogenous disturbance belonging to $l_{2}[0, T]$, and $w_{k}$ is a zero-mean scalar Gaussian random sequence with variance $\mathbf{E}\left\{w_{k}^{2}\right\}=1$. $B_{i, k}$ is a timevarying matrix with appropriate dimensions. $r_{i j}(i, j \in \mathcal{N})$ is the outer coupling between two nodes $i$ and $j . r_{i j}>0(j \neq i)$ indicates that there is information transmission from the node $j$ to node $i$; otherwise $r_{i j}=0$. We assume in this paper that the information transmission is symmetric and equivalent between two different nodes, i.e., $r_{i j}=r_{j i}$, and the diffusive condition $r_{i i}+\sum_{j=1, j \neq i}^{N} r_{i j}=0$ is satisfied.

The nonlinear vector functions $f\left(x_{i, k}\right) \in \mathbb{R}^{n}$ and $h_{i}\left(x_{i, k}\right) \in$ $\mathbb{R}^{n}$ satisfy the following assumption.

Assumption 1: For any vectors $\mu_{k}, \nu_{k} \in \mathbb{R}^{n}$, the followings are true:

$$
\begin{aligned}
& {\left[f\left(\mu_{k}\right)-f\left(\nu_{k}\right)-F_{1, k}\left(\mu_{k}-\nu_{k}\right)\right]^{T}} \\
& \quad \times\left[f\left(\mu_{k}\right)-f\left(\nu_{k}\right)-F_{2, k}\left(\mu_{k}-\nu_{k}\right)\right] \leq 0 \\
& \left\|h_{i}\left(\mu_{k}\right)-h_{i}\left(\nu_{k}\right)\right\|^{2} \leq\left\|S_{i, k}\left(\mu_{k}-\nu_{k}\right)\right\|^{2}
\end{aligned}
$$

where $f(0)=0, h_{i}(0)=0$, and $F_{1, k}, F_{2, k}, S_{i, k}$ are known matrices.

The inner coupling of the coupled network (1) is

$$
\begin{aligned}
G_{k} & =\operatorname{diag}\left\{\psi_{1, k}, \cdots, \psi_{n, k}\right\} \cdot \operatorname{diag}\left\{g_{1}, \cdots, g_{n}\right\} \\
& \triangleq \Psi_{k} G_{0}
\end{aligned}
$$

where $g_{q}>0(q=1,2, \cdots n)$ are known scalars, and $\psi_{q, k}$ are mutually independent random sequences distributed over the intervals $\left[\underline{u}_{q}, \bar{u}_{q}\right]$ with known scalars $\bar{u}_{q} \geq \underline{u}_{q} \geq 0$. The mathematical expectations and variances of $\psi_{q, k}$ are $\psi_{q}$ and $\sigma_{q}^{2}$, respectively. Correspondingly, the expectation of the inner coupling matrix $G_{k}$ is

$$
G=\mathbf{E}\left\{G_{k}\right\}=\Psi G_{0}=\operatorname{diag}\left\{\psi_{1} g_{1}, \cdots, \psi_{n} g_{n}\right\} .
$$

Assumption 2: The random sequences $w_{k}$ and $\psi_{q, k}$ are mutually independent. 
Remark 1: Both the stochastic noise $w(k)$ and $l_{2}$-type external disturbance $v(k)$ are considered in this paper. In contrast with most existing literature, the inner coupling strength matrix $G_{k}$ in (1) is allowed to change randomly. As pointed out in [22], the inner couplings cannot be exactly known in many real-world coupled networks due to unavoidable variations of the inner connection among subsystems. In fact, the inner connection coefficients of the practical coupled networks are mainly identified through statistic methods and/or measurement technologies, and this would inevitably bring some kind of stochastic perturbations. Furthermore, different from most existing results (see e.g. [22]), the model (3) ensures the inner coupling strengths to be nonnegative and bounded, which reflects the engineering practice closely.

\section{B. Round-Robin Protocol and Estimation Error Dynamics}

For the addressed complex network (1), the measurement of the sensor $i$ is described by

$$
\tilde{y}_{i, k}=C_{i, k} x_{i, k}+D_{i, k} v_{k}
$$

where $\tilde{y}_{i, k} \in \mathbb{R}^{n_{y}}, v_{k} \in \mathbb{R}^{n_{v}}$ is the disturbance as specified in (1), and $C_{i, k}, D_{i, k}(i \in \mathcal{N})$ are time-varying matrices with compatible dimensions.

In this paper, the measurement signals are transmitted through a non-ideal communication channel with limited bandwidth. In order to alleviate the network load and avoid possible network congestion, the RRP is applied to schedule the signal transmission. Based on the RRP, at each time instant, only one node has the access to transmit its information through the shared communication channel. Thus, the real measurement received by the estimator $i$ is

$$
y_{i, k}= \begin{cases}\tilde{y}_{i, k}, & \bmod (k, N)=i \\ y_{i, k-1}, & \text { otherwise }, i \in \mathcal{N}\end{cases}
$$

with the initial condition $y_{i, 0}=0$.

Denote

$$
y_{k} \triangleq\left[\begin{array}{lll}
y_{1, k}^{T} & y_{2, k}^{T} \cdots y_{N, k}^{T}
\end{array}\right]^{T}, \quad \tilde{y}_{k} \triangleq\left[\begin{array}{lll}
\tilde{y}_{1, k}^{T} & \tilde{y}_{2, k}^{T} \cdots \tilde{y}_{N, k}^{T}
\end{array}\right]^{T} .
$$

Hence, the measurement updated equation is written as

$$
y_{k}=I_{\sigma_{k}} \tilde{y}_{k}+\left(I-I_{\sigma_{k}}\right) y_{k-1}
$$

where

$$
I_{\sigma_{k}}=\operatorname{diag}\{\underbrace{0, \cdots, 0}_{\sigma_{k}-1}, I, \underbrace{0, \cdots, 0}_{N-\sigma_{k}}\}
$$

with $\sigma_{k} \in \mathcal{N}$ and $\sigma_{k}=\bmod (k-1, N)+1$.

Based on (6), the Luenberger-type state estimator for each node is constructed as

$$
\hat{x}_{i, k+1}=f\left(\hat{x}_{i, k}\right)+\sum_{j=1}^{N} r_{i j} G \hat{x}_{j, k}+L_{i, k}\left(y_{i, k}-C_{i, k} \hat{x}_{i, k}\right)
$$

where $\hat{x}_{i, k}$ is the estimate of $x_{i, k}$, and $L_{i, k}$ is the time-varying gain matrix to be designed.

By defining the estimation error of the $i$-th estimator as $e_{i, k} \triangleq x_{i, k}-\hat{x}_{i, k}$, and denoting $x_{k} \triangleq\left[x_{1, k}^{T} x_{2, k}^{T} \cdots x_{N, k}^{T}\right]^{T}$, $\hat{x}_{k} \triangleq\left[\hat{x}_{1, k}^{T} \hat{x}_{2, k}^{T} \cdots \hat{x}_{N, k}^{T}\right]^{T}$ and $e_{k} \triangleq\left[e_{1, k}^{T} e_{2, k}^{T} \cdots e_{N, k}^{T}\right]^{T}$, the estimator (8) can be written as

$$
\hat{x}_{k+1}=F_{k, \hat{x}}+(R \otimes G) \hat{x}_{k}+L_{k}\left(y_{k}-C_{k} \hat{x}_{k}\right)
$$

where

$$
\begin{aligned}
F_{k, \hat{x}} & \triangleq\left[f^{T}\left(\hat{x}_{1, k}\right) f^{T}\left(\hat{x}_{2, k}\right) \cdots f^{T}\left(\hat{x}_{N, k}\right)\right]^{T} \\
R & \triangleq\left[r_{i j}\right]_{N \times N} \\
L_{k} & \triangleq \operatorname{diag}\left\{L_{1, k}, L_{2, k}, \cdots, L_{N, k}\right\} \\
C_{k} & \triangleq \operatorname{diag}\left\{C_{1, k}, C_{2, k}, \cdots, C_{N, k}\right\} .
\end{aligned}
$$

By setting $f\left(\chi_{i, k}\right)=f\left(x_{i, k}\right)-f\left(\hat{x}_{i, k}\right)$ and noticing (1), (7) and (8), we have

$$
\begin{aligned}
e_{k+1}= & F_{k, e}+\left[R \otimes\left(G_{k}-G\right)+L_{k}\left(I-I_{\sigma_{k}}\right) C_{k}\right] x_{k} \\
& +\left(R \otimes G-L_{k} C_{k}\right) e_{k}-L_{k}\left(I-I_{\sigma_{k}}\right) y_{k-1} \\
& +\left(B_{k}-L_{k} I_{\sigma_{k}} D_{k}\right) v_{k}+H_{k, x} w_{k}
\end{aligned}
$$

where

$$
\begin{aligned}
F_{k, e} & \triangleq\left[f^{T}\left(\chi_{1, k}\right) f^{T}\left(\chi_{2, k}\right) \cdots f^{T}\left(\chi_{N, k}\right)\right]^{T} \\
B_{k} & \triangleq\left[B_{1, k}^{T}, B_{2, k}^{T}, \cdots, B_{N, k}^{T}\right]^{T} \\
D_{k} & \triangleq\left[D_{1, k}^{T}, D_{2, k}^{T}, \cdots, D_{N, k}^{T}\right]^{T} \\
H_{k, x} & \triangleq\left[h_{1}^{T}\left(x_{1, k}\right) h_{2}^{T}\left(x_{2, k}\right) \cdots h_{N}^{T}\left(x_{N, k}\right)\right]^{T} .
\end{aligned}
$$

Let the output signal (to be estimated) for the underlying complex network (1) be given by

$$
z_{i, k}=E_{i, k} x_{i, k}
$$

where the time-varying matrix $E_{i, k}$ is dimensionally compatible. Then, one obtains

$$
\begin{aligned}
\bar{z}_{i, k} & \triangleq z_{i, k}-\hat{z}_{i, k}=E_{i, k} e_{i, k} \\
& =E_{i, k} x_{i, k}-E_{i, k} \hat{x}_{i, k} .
\end{aligned}
$$

Setting $\eta_{k} \triangleq\left[\begin{array}{lll}x_{k}^{T} & y_{k-1}^{T} & e_{k}^{T}\end{array}\right]^{T}, \bar{z}_{k} \triangleq z_{k}-\hat{z}_{k}, z_{k} \triangleq$ $\left[\begin{array}{ll}z_{1, k}^{T} & z_{2, k}^{T} \cdots z_{N, k}^{T}\end{array}\right]^{T}$ and $\hat{z}_{k} \triangleq\left[\begin{array}{ll}\hat{z}_{1, k}^{T} & \hat{z}_{2, k}^{T} \cdots \hat{z}_{N, k}^{T}\end{array}\right]^{T}$, the dynamics of $\eta_{k}$ is expressed as

$$
\left\{\begin{aligned}
\eta_{k+1} & =\left(\mathcal{A}_{k}+\Delta_{k}\right) \eta_{k}+\mathcal{F}_{k}+\mathcal{B}_{k} v_{k}+\mathcal{H}_{k} w_{k} \\
\bar{z}_{k} & =\mathcal{E}_{k} \eta_{k}
\end{aligned}\right.
$$

where

$$
\begin{aligned}
& \mathcal{A}_{k} \triangleq {\left[\begin{array}{ccc}
R \otimes G & 0 & 0 \\
I_{\sigma_{k}} C_{k} & I_{\sigma_{k}}^{\delta} & 0 \\
L_{k} I_{\sigma_{k}}^{\delta} C_{k} & -L_{k} I_{\sigma_{k}}^{\delta} & R \otimes G-L_{k} C_{k}
\end{array}\right] } \\
& \Delta_{k} \triangleq\left[\begin{array}{ccc}
R \otimes\left(G_{k}-G\right) & 0 & 0 \\
0 & 0 & 0 \\
R \otimes\left(G_{k}-G\right) & 0 & 0
\end{array}\right] \\
& \mathcal{F}_{k} \triangleq\left[\begin{array}{c}
F_{k, x} \\
0 \\
F_{k, e}
\end{array}\right], \mathcal{H}_{k} \triangleq\left[\begin{array}{c}
H_{k, x} \\
0 \\
H_{k, x}
\end{array}\right] \\
& \mathcal{B}_{k} \triangleq\left[\begin{array}{c}
B_{k} \\
I_{\sigma_{k}} D_{k} \\
B_{k}-L_{k} D_{k}
\end{array}\right] \\
& \mathcal{E}_{k} \triangleq\left[\begin{array}{cc}
0 & E_{k}
\end{array}\right] \\
& E_{k} \triangleq \operatorname{diag}\left\{E_{1, k}, E_{2, k}, \cdots, E_{N, k}\right\} \\
& I_{\sigma_{k}}^{\delta} \triangleq I-I_{\sigma_{k}} .
\end{aligned}
$$


Remark 2: The RRP is introduced in this paper to ease the network load and avoid the possible network-induced problems in the shared communication channel. Due to the introduction of $I_{\sigma_{k}}$, the measurement equation (7) and the augmented system (12) exhibit the periodically switching behaviors. However, different from the standard switched systems [5], [7], [37], the switching caused by $I_{\sigma_{k}}$ in system (12) is $N$-periodic in a fixed circle with a constant switching time interval.

\section{Approximations of Nonlinear Functions $f(\cdot)$ and $h_{i}(\cdot)$}

It is observed from (12) that two nonlinear vector-valued functions $\mathcal{F}_{k}$ and $\mathcal{H}_{k}$ correspond to the nonlinear functions $f(\cdot)$ and $h_{i}(\cdot)$, respectively. In order to design the $H_{\infty}$ estimator (8) by means of Riccati-type difference equation method, the nonlinear vector functions $\mathcal{F}_{k}$ and $\mathcal{H}_{k}$ are now handled properly as in [9], [21].

Let us consider a time-varying nonlinear scalar-valued function $c\left(\nu_{k}\right)$ satisfying $\left[c\left(\nu_{k}\right)-a \nu_{k}\right]\left[c\left(\nu_{k}\right)+a \nu_{k}\right] \leq 0$, which is equivalent to $c^{2}\left(\nu_{k}\right) \leq a^{2} \nu_{k}^{2}$ or $-a \nu_{k} \leq c\left(\nu_{k}\right) \leq a \nu_{k}$, where $a>0$ is a known scalar. It is easy to verify that there indeed exists a scalar $m_{k} \in[-1,1]$ such that $c\left(\nu_{k}\right)=m_{k} \cdot a \nu_{k}$ for any $k=0,1,2, \cdots$. Note that the above technical handling process can be directly applied to the nonlinear vector-valued function $f\left(x_{i, k}\right)$ subject to $(2 \mathrm{a})$.

For the nonlinear vector-valued function $f\left(x_{i, k}\right)$ with $f(0)=0$, the inequality $(2 \mathrm{a})$ can be rewritten as

$$
\begin{aligned}
& {\left[\left(f\left(x_{i, k}\right)-\Theta_{k} x_{i, k}\right)-\Upsilon_{k} x_{i, k}\right]^{T}} \\
& \quad \times\left[\left(f\left(x_{i, k}\right)-\Theta_{k} x_{i, k}\right)+\Upsilon_{k} x_{i, k}\right] \leq 0
\end{aligned}
$$

where

$$
\Theta_{k}=\frac{F_{1, k}+F_{2, k}}{2}, \quad \Upsilon_{k}=\frac{F_{1, k}-F_{2, k}}{2} .
$$

Similar to the scalar case discussed previously, there always exists a matrix $M_{k}$ satisfying $M_{k}^{T} M_{k} \leq I$ such that $f\left(x_{i, k}\right)-$ $\Theta_{k} x_{i, k}=M_{k} \Upsilon_{k} x_{i, k}$ or

$$
f\left(x_{i, k}\right)=\Theta_{k} x_{i, k}+M_{k} \Upsilon_{k} x_{i, k}
$$

Also, one has $f\left(\chi_{i, k}\right)=\Theta_{k} e_{i, k}+M_{k} \Upsilon_{k} e_{i, k}$. In view of (2b), the nonlinear function $h_{i}\left(x_{i, k}\right)$ can now be rearranged as

$$
h_{i}\left(x_{i, k}\right)=\bar{M}_{k} S_{i, k} x_{i, k}
$$

where the matrix $\bar{M}_{k}$ satisfies $\bar{M}_{k}^{T} \bar{M}_{k} \leq I$.

By introducing the following notations

$$
\begin{aligned}
& \digamma_{k} \triangleq \operatorname{diag}\{\underbrace{\Theta_{k}, \Theta_{k}, \cdots, \Theta_{k}}_{N}\} \\
& \mathfrak{F}_{k}=\operatorname{diag}\{\underbrace{\Upsilon_{k}, \Upsilon_{k}, \cdots, \Upsilon_{k}}_{N}\} \\
& S_{k} \triangleq \operatorname{diag}\left\{\left\{S_{1, k}, S_{2, k}, \cdots, S_{N, k}\right\}\right. \\
& \mathbf{F}_{k} \triangleq \operatorname{diag}\left\{\digamma_{k}, 0, \digamma_{k}\right\} \\
& \mathbb{F}_{k} \triangleq \operatorname{diag}\left\{\mathfrak{F}_{k}, 0, \mathfrak{F}_{k}\right\} \\
& \mathbb{H}_{k} \triangleq\left[\begin{array}{lll}
S_{k}^{T} & 0 & S_{k}^{T}
\end{array}\right]^{T}
\end{aligned}
$$

the nonlinear functions $\mathcal{F}_{k}$ and $\mathcal{H}_{k}$ in (12) are expressed as

$$
\begin{aligned}
\mathcal{F}_{k} & =\left(\mathbf{F}_{k}+M_{k} \mathbb{F}_{k}\right) \eta_{k} \\
\mathcal{H}_{k} & =\bar{M}_{k} \mathbb{H}_{k} x_{k} .
\end{aligned}
$$

Rearranging (12) gives the following auxiliary equation

$$
\begin{aligned}
\eta_{k+1} & =\left(\mathcal{A}_{k}+\mathbf{F}_{k}+\Delta_{k}\right) \eta_{k}+\mathbf{B}_{k} \mathbf{v}_{k} \\
\bar{z}_{k} & =\mathcal{E}_{k} \eta_{k}
\end{aligned}
$$

where $\mathcal{A}_{k}, \Delta_{k}, \mathcal{E}_{k}$ and $\mathbf{F}_{k}$ are given in (13) and (16), respectively, and

$$
\begin{aligned}
\mathbf{B}_{k} & \triangleq\left[\begin{array}{lll}
\mathcal{B}_{k} & \alpha_{k}^{-1} I & \beta_{k}^{-1} I w_{k}
\end{array}\right] \\
\mathbf{v}_{k} & \triangleq\left[\begin{array}{lll}
v_{k}^{T} & \alpha_{k}\left(M_{k} \mathbb{F}_{k} \eta_{k}\right)^{T} & \beta_{k}\left(\bar{M}_{k} \mathbf{H}_{k} \eta_{k}\right)^{T}
\end{array}\right]^{T} \\
\mathbf{H}_{k} & \triangleq\left[\begin{array}{lll}
\mathbb{H}_{k} & 0 & 0
\end{array}\right]
\end{aligned}
$$

with $\alpha_{k}, \beta_{k}$ being tuning scalars to enhance the feasibility of the addressed estimator design problem.

Remark 3: The two nonlinear vector-valued functions $f(\cdot)$ and $h_{i}(\cdot)$ are transformed to (14) and (15), respectively, based on which the linear difference equation (17) with time-varying parametric uncertainties is obtained. It is noted that (15) is a special case of (14) with $F_{1, k}=-F_{2, k}$, in which the nonlinear function $f\left(x_{i, k}\right)$ is decomposed into two parts, i.e., the certain term $\Theta_{k} x_{i, k}$ and the uncertain term $M_{k} \Upsilon_{k} x_{i, k}$, respectively.

\section{Main Objective and Preliminary Results}

In this paper, we aim to construct the state estimator (9) for the network (1) with the random inner coupling (3) and the Round-Robin scheduling protocol (6) such that the resulting augmented estimation error dynamics (12) satisfies the following $H_{\infty}$ performance index:

$$
J_{1}=\sum_{k=0}^{T} \mathbf{E}\left\{\left\|\bar{z}_{k}\right\|^{2}-\gamma^{2}\left\|v_{k}\right\|^{2}-\gamma^{2} \eta_{0}^{T} W \eta_{0}\right\}<0
$$

for a prescribed disturbance attenuation level $\gamma>0$ over a finite time horizon $[0, T]$, where $W>0$ is a weighted matrix and $\eta_{0}$ is any given nonzero initial condition.

For the auxiliary system (17), the following performance index is defined

$$
\begin{array}{rl}
J_{2}=\sum_{k=0}^{T} & \mathbf{E}\left\{\left\|\bar{z}_{k}\right\|^{2}-\gamma^{2}\left(\left\|\mathbf{v}_{k}\right\|^{2}-\left\|\alpha_{k} \mathbb{F}_{k} \eta_{k}\right\|^{2}\right.\right. \\
& \left.\left.-\left\|\beta_{k} \mathbf{H}_{k} \eta_{k}\right\|^{2}\right)-\gamma^{2} \eta_{0}^{T} W \eta_{0}\right\}
\end{array}
$$

From the definitions of $J_{1}, J_{2}, \mathbf{v}_{k}$, it is clear that

$$
\begin{array}{r}
J_{1}-J_{2}=\sum_{k=0}^{T} \mathbf{E}\left\{\gamma^{2}\left(\left\|\alpha_{k} M_{k} \mathbb{F}_{k} \eta_{k}\right\|^{2}-\left\|\alpha_{k} \mathbb{F}_{k} \eta_{k}\right\|^{2}\right)\right. \\
\left.+\gamma^{2}\left(\left\|\beta_{k} \bar{M}_{k} \mathbf{H}_{k} \eta_{k}\right\|^{2}-\left\|\beta_{k} \mathbf{H}_{k} \eta_{k}\right\|^{2}\right)\right\} .
\end{array}
$$

By employing $M_{k}^{T} M_{k} \leq I$ and $\bar{M}_{k}^{T} \bar{M}_{k} \leq I$, we obtain

$$
\begin{array}{rl}
J_{1}-J_{2} \leq \sum_{k=0}^{T} & \mathbf{E}\left\{\gamma^{2}\left(\left\|M_{k}\right\|^{2}-I\right)\left\|\alpha_{k} \mathbb{F}_{k} \eta_{k}\right\|^{2}\right. \\
& \left.+\gamma^{2}\left(\left\|\bar{M}_{k}\right\|^{2}-I\right)\left\|\beta_{k} \mathbf{H}_{k} \eta_{k}\right\|^{2}\right\} \leq 0
\end{array}
$$


which means that $J_{1}<0$ is ensured by $J_{2}<0$. Hence, in the next section we will concentrate on designing an estimator (8) such that $J_{2}<0$.

Before proceeding further, the following two lemmas are presented.

Lemma 1: ( [13]) Let $P=\left[p_{i j}\right]_{n \times n}$ be a real matrix and $R=\operatorname{diag}\left\{r_{1}, r_{2}, \cdots, r_{n}\right\}$ be a diagonal stochastic matrix. Then, we have

$\mathbf{E}\left\{R^{T} P R\right\}=\left[\begin{array}{cccc}\mathbf{E}\left\{r_{1}^{2}\right\} & \mathbf{E}\left\{r_{1} r_{2}\right\} & \cdots & \mathbf{E}\left\{r_{1} r_{n}\right\} \\ \mathbf{E}\left\{r_{2} r_{1}\right\} & \mathbf{E}\left\{r_{2}^{2}\right\} & \cdots & \mathbf{E}\left\{r_{2} r_{n}\right\} \\ \vdots & \vdots & \ddots & \vdots \\ \mathbf{E}\left\{r_{n} r_{1}\right\} & \mathbf{E}\left\{r_{n} r_{2}\right\} & \cdots & \mathbf{E}\left\{r_{n}^{2}\right\}\end{array}\right] \circ P$

where $\circ$ is the Hadamard product.

Lemma 2: ( [2]) For any zero-mean scalar Gaussian random sequence $w_{k}$ with prior variance $\mathbf{E}\left\{w_{k}^{2}\right\}=\sigma^{2}$, the following holds:

$$
\mathbf{E}\left\{w_{k}^{2} w_{l}^{2}\right\}= \begin{cases}\sigma^{4}, & \text { if } k \neq l \\ 3 \sigma^{4}, & \text { if } k=l .\end{cases}
$$

\section{MAIN RESULTS}

In this section, a criterion is established to ensure the finite-horizon $H_{\infty}$ performance constraint $J_{2}<0$ for the auxiliary system (17) based on the BCRRDE approach. Then, the estimator (8) is determined by solving two BCRRDEs. Moreover, a recursive algorithm is provided to compute the gain matrix of the estimator (8).

For notational simplicity, we denote:

$$
\begin{aligned}
& \mathbb{A}_{k} \triangleq \mathcal{A}_{k}+\mathbf{F}_{k} \\
& \Xi_{k} \triangleq\left[\begin{array}{lll}
\mathcal{B}_{k} & \alpha_{k}^{-1} I & 0
\end{array}\right] \\
& \Gamma_{k} \triangleq\left[\begin{array}{lll}
0 & 0 & \beta_{k}^{-1} I
\end{array}\right] \\
& X_{k} \triangleq \Xi_{k}^{T} P_{k+1} \Xi_{k}+\Gamma_{k}^{T} P_{k+1} \Gamma_{k} \\
& Y_{k} \triangleq \Xi_{k} \Omega_{k}^{-1} \Xi_{k}^{T}+\Gamma_{k} \Omega_{k}^{-1} \Gamma_{k}^{T} \\
& \mathbf{M}_{k} \triangleq P_{k+1}\left(Y_{k} Q_{k+1} Y_{k}+4 \Xi_{k} \Omega_{k}^{-1} \Gamma_{k}^{T} Q_{k+1} \Gamma_{k} \Omega_{k}^{-1} \Xi_{k}^{T}\right. \\
& \left.+2 \Gamma_{k} \Omega_{k}^{-1} \Gamma_{k}^{T} Q_{k+1} \Gamma_{k} \Omega_{k}^{-1} \Gamma_{k}^{T}\right) P_{k+1} \\
& Z_{k} \triangleq Q_{k+1}+Q_{k+1} Y_{k} P_{k+1}+P_{k+1} Y_{k} Q_{k+1}+\mathbf{M}_{k} \\
& \overline{\mathbf{G}} \triangleq\left[\begin{array}{ccc}
R \otimes \bar{G} & 0 & 0 \\
0 & 0 & 0 \\
R \otimes \bar{G} & 0 & 0
\end{array}\right] \\
& \bar{G} \triangleq G_{0} \operatorname{diag}\left\{\sigma_{1}, \sigma_{2}, \cdots, \sigma_{n}\right\} \\
& \phi_{k} \triangleq L_{k} \mathbf{C}_{k} \eta_{k} \\
& \mathcal{I} \triangleq\left[\begin{array}{lll}
0 & 0 & I
\end{array}\right]^{T} \\
& \mathbf{C}_{k} \triangleq\left[\begin{array}{lll}
0 & 0 & -C_{k}
\end{array}\right] \text {. }
\end{aligned}
$$

\section{A. Estimator Performance Analysis}

For the auxiliary system (17), the following theorem is established.

Theorem 1: Consider the coupled network (1) with fixed estimator (8), where the measurement is given by (7). For a given scalar $\gamma>0$ and a weighted matrix $W>0$, the auxiliary system (17) achieves the prescribed $H_{\infty}$ disturbance rejection level $\gamma$ if there exist a set of positive definite matrices $P_{k}$ and two sets of positive scalars $\alpha_{k}, \beta_{k}(k=0,1,2, \cdots, T)$ such that the following backward Riccati recursive equations

$$
\begin{aligned}
P_{k}= & \mathbb{A}_{k}^{T} P_{k+1} \mathbb{A}_{k}+\overline{\mathbf{G}}^{T} P_{k+1} \overline{\mathbf{G}}+\mathcal{E}_{k}^{T} \mathcal{E}_{k}+\gamma^{2} \alpha_{k}^{2} \mathbb{F}_{k}^{T} \mathbb{F}_{k} \\
& +\gamma^{2} \beta_{k}^{2} \mathbf{H}_{k}^{T} \mathbf{H}_{k}+\mathbb{A}_{k}^{T} P_{k+1} \Xi_{k} \Omega_{k}^{-1} \Xi_{k}^{T} P_{k+1} \mathbb{A}_{k}
\end{aligned}
$$

are feasible with $P_{T+1}=0$ and

$$
\begin{aligned}
& \Omega_{k}=\gamma^{2} I-X_{k}>0 \\
& P_{0}<\gamma^{2} W .
\end{aligned}
$$

Proof: See Appendix.

Remark 4: From the proof of Theorem 1, we can see that the random inner coupling matrix $G_{k}$ and the state-dependent multiplicative stochastic noise $h_{i}\left(x_{i, k}\right) w_{k}$ pose substantial difficulties on the analysis of the $H_{\infty}$ performance. By utilizing Lemma 1, the randomness of the matrix $G_{k}$ is eventually reflected by the term $\overline{\mathbf{G}}^{T} P_{k+1} \overline{\mathbf{G}}$ in (21). By the decomposition operation in (34), the effects of stochastic noise are reflected by both the last term in (21) and $X_{k}$ in (22).

We now proceed to design the finite-horizon $H_{\infty}$ estimator (8) for the network (1) under the worst-case disturbances $\mathbf{v}_{k}^{*}$.

Replacing $\mathbf{v}_{k}$ by $\mathbf{v}_{k}^{*}=\Omega_{k}^{-1} \mathbf{B}_{k}^{T} P_{k+1}\left(\mathcal{A}_{k}+\mathbf{F}_{k}\right) \eta_{k}$ in (17) and decomposing $\mathcal{A}_{k}$ into $\mathcal{A}_{k}=\overline{\mathcal{A}}_{k}+\mathcal{I} L_{k} \mathbf{C}_{k}$, we have

$$
\begin{aligned}
\eta_{k+1} & =\left(\overline{\mathbb{A}}_{k}+\overline{\mathbb{B}}_{k} \overline{\mathbb{A}}_{k}+\Delta_{k}\right) \eta_{k}+\left(\overline{\mathbb{B}}_{k}+I\right) \mathcal{I} \phi_{k} \\
\bar{z}_{k} & =\mathcal{E}_{k} \eta_{k}
\end{aligned}
$$

where

$$
\begin{aligned}
& \overline{\mathbb{A}}_{k} \triangleq \overline{\mathcal{A}}_{k}+\mathbf{F}_{k} \\
& \overline{\mathcal{A}}_{k} \triangleq\left[\begin{array}{ccc}
R \otimes G & 0 & 0 \\
I_{\sigma_{k}} C_{k} & I_{\sigma_{k}}^{\delta} & 0 \\
L_{k} I_{\sigma_{k}}^{\delta} C_{k} & -L_{k} I_{\sigma_{k}}^{\delta} & R \otimes G
\end{array}\right] \\
& \mathbb{B}_{k} \triangleq \overline{\mathbb{B}}_{k} \overline{\mathbb{A}}_{k}+\mathcal{I} L_{k} \mathbf{C}_{k} \\
& \overline{\mathbb{B}}_{k} \triangleq \mathbf{B}_{k} \Omega_{k}^{-1} \mathbf{B}_{k}^{T} P_{k+1} \\
&=\left(\Xi_{k} \Omega_{k}^{-1} \Xi_{k}^{T}+2 \Xi_{k} \Omega_{k}^{-1} \Gamma_{k}^{T} w_{k}+\Gamma_{k} \Omega_{k}^{-1} \Gamma_{k}^{T} w_{k}^{2}\right) P_{k+1} .
\end{aligned}
$$

Define the following performance index for system (23)

$$
\tilde{J} \triangleq \sum_{k=0}^{T} \mathbf{E}\left\{\left\|\bar{z}_{k}\right\|^{2}+\left\|\phi_{k}\right\|\right\} .
$$

In the sequel, we will construct the estimator (8) by minimizing the index $\tilde{J}$ in (25). The corresponding result is presented as follows.

Theorem 2: Consider the coupled network (1) with the measurement (7). Assume that there are two sets of positive definite matrices $P_{k}, Q_{k}$ and two sets of positive scalars $\alpha_{k}, \beta_{k}$ $(k=0,1,2, \cdots, T)$ such that, for a given positive scalar $\gamma>0$ and a weighted matrix $W>0$, the coupled backward Riccati difference equations (21) and

$$
\begin{aligned}
Q_{k}= & \overline{\mathbb{A}}_{k}^{T} Z_{k} \overline{\mathbb{A}}_{k}+\overline{\mathbf{G}}^{T} Q_{k+1} \overline{\mathbf{G}}+\mathcal{E}_{k}^{T} \mathcal{E}_{k}+\overline{\mathbb{A}}_{k}^{T}\left(\mathbf{M}_{k}\right. \\
& \left.+Q_{k+1} Y_{k} P_{k+1}\right) \mathcal{I} L_{k} \mathbf{C}_{k}+\mathbf{C}_{k}^{T} L_{k}^{T} \mathcal{I}^{T}\left(\mathbf{M}_{k}\right. \\
& \left.+P_{k+1} Y_{k} Q_{k+1}\right) \overline{\mathbb{A}}_{k}-\overline{\mathbb{A}}_{k}^{T}\left(P_{k+1} Y_{k}+I\right) \\
& \times Q_{k+1} \mathcal{I} \Psi_{k}^{-1} \mathcal{I}^{T} Q_{k+1}\left(Y_{k} P_{k+1}+I\right) \overline{\mathbb{A}}_{k}
\end{aligned}
$$

are feasible under the conditions $P_{T+1}=0, Q_{T+1}=0$, (22) and

$$
\Psi_{k}=\mathcal{I}^{T} Z_{k} \mathcal{I}+I>0
$$


Then, (8) is the $H_{\infty}$ estimator of (1) satisfying

$$
L_{k} \mathbf{C}_{k}=-\Psi_{k}^{-1} \mathcal{I}^{T} Q_{k+1}\left(Y_{k} P_{k+1}+I\right) \overline{\mathbb{A}}_{k} .
$$

The values of the given cost functions $J_{2}$ and $\tilde{J}$ are, respectively, given as

$$
J_{2}=\eta_{0}^{T}\left(P_{0}-\gamma^{2} W\right) \eta_{0}, \tilde{J}=\eta_{0}^{T} Q_{0} \eta_{0} .
$$

Proof: See Appendix.

Remark 5: It should be noted that, since the augmented disturbance vector $\mathbf{v}_{k}$ in (18) does not explicitly contain the Gaussian noise $w_{k}$ which actually exists in $\mathbf{B}_{k}$, it is reasonable to require that the worst-case disturbance is set as $\mathbf{v}_{k} \triangleq \mathbf{v}_{k}^{*}=\Omega_{k}^{-1} \mathbf{B}_{k}^{T} P_{k+1}\left(\mathcal{A}_{k}+\mathbf{F}_{k}\right) \eta_{k}$. In fact, (17) can be viewed as a kind of stochastic parameter systems which have been investigated in the existing literature [8].

\section{B. Estimator Design}

In this subsection, we will present an algorithm to compute the estimator gain matrix $L_{i, k}$ in (8).

For brevity, the notations

$$
\begin{gathered}
W_{k} \triangleq-\Psi_{k}^{-1} \mathcal{I}^{T} Q_{k+1}\left(Y_{k} P_{k+1}+I\right) \overline{\mathbb{A}}_{k} \\
\tilde{I}_{i} \triangleq \operatorname{diag}\{\underbrace{0, \cdots, 0}_{i-1}, I_{n \times n}, \underbrace{0, \cdots, 0}_{N-i}\}
\end{gathered}
$$

are adopted In the sequel.

Noticing the form of $\mathbf{C}_{k}$ in (20), the following equation

$$
L_{k} C_{k}=\bar{W}_{k}
$$

holds, where $\bar{W}_{k} \in \mathbb{R}^{N n \times N n}$ is the third block element of matrix $W_{k}$. Moreover, it can be found that $L_{i, k} C_{i, k}=\bar{W}_{k} \tilde{I}_{i}$. Hence, the gain matrix of estimator (8) is computed by using Moore-Penrose pseudo inverse, which is stated in the following Theorem.

Theorem 3: Consider the coupled network (1) the measurement (7). For a given scalar $\gamma>0$ and a weighted matrix $W>0$, if there are two sets of positive definite matrices $P_{k}, Q_{k}$ with $P_{T+1}=0, Q_{T+1}=0$, and two sets of positive scalars $\alpha_{k}, \beta_{k}(k=0,1,2, \cdots, T)$ such that the coupled backward Riccati difference equations (21) and (26) hold under the constraints (22) and (27), then (8) is the $H_{\infty}$ estimator of (1) with the gain matrix given by

$$
L_{i, k}=\bar{W}_{k} \tilde{I}_{i} C_{i, k}^{\dagger} .
$$

To facilitate the implementation, the following finite-horizon $H_{\infty}$ estimator (FHHE) design algorithm is provided to recursively compute the gain matrix $L_{i, k}$ based on Theorems 1-3.

Remark 6: In this paper, the problem of finite-horizon $H_{\infty}$ state estimation is dealt with for a class of time-varying coupled stochastic networks under the Round-Robin scheduling protocol. The underlying network model is quite comprehensive that features stochastic inner coupling strengths and Round-Robin protocol scheduling. Some dedicated approximation techniques are used to establish an uncertain auxiliary system whose coefficient matrix is subject to multiplicative noises, thereby giving rise to a certain stochastic parameter

\section{Algorithm FHHE}

Step 1. Give the scalars $\gamma>0, \alpha_{k}, \beta_{k}(k=0,1, \cdots, T)$, weighted
matrix $W>0$, and let $k=T$ and $P_{T+1}=Q_{T+1}=0$.
Step 2. Calculate the matrix $\Omega_{k}$ by $(22)$, and solve $(27)$ to obtain the
matrix $\Psi_{k}$. If $\Psi_{k}>0$, then $L_{i, k}$ can be determined by (30),
and go to the next step, otherwise go to step 1.
Step 3. If $\Omega_{k}>0$, then $P_{k}$ and $Q_{k}$ can be obtained by (21) and (26),
respectively, and then go to the next step, else go to step 5.
Step 4. If $k>0$, set $k=k-1$ and go to step 2 . When $k=0$, stop.
Step 5. If anyone in (22) and (27) is violated, then this algorithm is
infeasible for given $\gamma, \alpha_{k}, \beta_{k}$ and $W$, stop or go to step 1
and start another recursive loop by resetting the values of $\gamma$,
$\alpha_{k}, \beta_{k}$ and $W$.

system. Based on such an auxiliary system, we go ahead to design the finite-horizon $H_{\infty}$ state estimator by solving coupled backward Riccati equations. A recursive algorithm is proposed to calculate the corresponding gain matrix of the $H_{\infty}$ estimator. Note that, in the conditions of our main results stated in Theorem 3, all the network information (e.g. the nonlinear functions, statistical law about the inner couplings, $H_{\infty}$ performance index) is reflected, which conforms with the engineering practice.

Remark 7: In this paper, a systematic investigation is initiated on the new yet challenging problem of RRP-based finite-horizon $H_{\infty}$ state estimation problem for a class of time-varying stochastic coupled networks subject to random inner coupling strengths. The main novelties of this paper are outlined as follows: 1) the research problem addressed is new that represents the first of few attempts to deal with the protocol-based state estimation problem for complex networks under hybrid phenomena of stochastic coupling, external disturbances and communication protocols; 2) the concept of finite-horizon $H_{\infty}$ performance is used to provide a reasonable way in evaluating the transient performance of the stochastic complex networks by means of the disturbance rejection/attenuation capacity; and 3) the developed BCRRE approach is new that offers a recursive algorithm suitable for online applications.

\section{An Illustrative Example}

This section provides an illustrative example to show the effectiveness of the state estimation method.

Consider the complex network (1) with three nodes $(N=3)$ over a prescribed finite time horizon $[0,40]$, i.e., $T=40$. The outer coupling configuration matrix is given as follows

$$
R=\left[\begin{array}{ccc}
-0.2 & 0.1 & 0.1 \\
0.1 & -0.2 & 0.1 \\
0.1 & 0.1 & -0.2
\end{array}\right]
$$

The time-varying nonlinear functions $f\left(x_{i, k}\right)$ and $h_{i}\left(x_{i, k}\right)$ are chosen as follows

$$
\begin{aligned}
f\left(x_{i, k}\right) & =\left[\begin{array}{c}
0.2 x_{i 1, k}+\tanh \left(0.1 x_{i 1, k}\right) \\
0.15 x_{i 2, k}+\tanh \left(0.05 x_{i 2, k}\right)
\end{array}\right] \\
h_{i}\left(x_{i, k}\right) & =\left[\begin{array}{c}
0.3 x_{i 1, k} \\
0.2 x_{i 2, k}+\tanh \left(0.1 x_{i 2, k}\right)
\end{array}\right]
\end{aligned}
$$


This article has been accepted for publication in a future issue of this journal, but has not been fully edited. Content may change prior to final publication. Citation information: DOI10.1109/TCYB.2020.3004288, IEEE Transactions on Cybernetics

TABLE I

ESTIMATOR GAIN MATRIX $L_{i, k}(i=1,2,3)$

\begin{tabular}{|c|c|c|c|}
\hline$k$ & $L_{1, k}$ & $L_{2, k}$ & $L_{3, k}$ \\
\hline 0 & $\begin{array}{l}-0.1445 \\
-0.0445\end{array}$ & $\begin{array}{c}0.0881 \\
-0.0044\end{array}$ & $\begin{array}{l}-0.2679 \\
-0.1960\end{array}$ \\
\hline 1 & $\begin{array}{l}-0.1436 \\
-0.0452\end{array}$ & $\begin{array}{c}0.0908 \\
-0.0066\end{array}$ & $\begin{array}{l}-0.2564 \\
-0.2037\end{array}$ \\
\hline 2 & $\begin{array}{l}-0.1421 \\
-0.0467\end{array}$ & $\begin{array}{c}0.0937 \\
-0.0086\end{array}$ & $\begin{array}{l}-0.2732 \\
-0.1901\end{array}$ \\
\hline$\vdots$ & $\vdots$ & $\vdots$ & $\vdots$ \\
\hline 38 & $\begin{array}{l}-0.1099 \\
-0.0694\end{array}$ & $\begin{array}{l}0.0488 \\
0.0240\end{array}$ & $\begin{array}{l}-0.2112 \\
-0.2183\end{array}$ \\
\hline 39 & $\begin{array}{l}-0.0859 \\
-0.0861\end{array}$ & $\begin{array}{l}0.0302 \\
0.0405\end{array}$ & $\begin{array}{l}-0.1610 \\
-0.2420\end{array}$ \\
\hline 40 & $\begin{array}{l}0 \\
0\end{array}$ & $\begin{array}{l}0 \\
0\end{array}$ & $\begin{array}{l}0 \\
0\end{array}$ \\
\hline
\end{tabular}

where $x_{i v, k}(v=1,2)$ is the $v$-th element of $x_{i, k}$, and the time-varying matrices $B_{i, k}(i=1,2,3)$ are given as follows

$$
\begin{aligned}
& B_{1, k}=\left[\begin{array}{c}
0.6+0.3 \sin (k) \\
-0.5
\end{array}\right] \\
& B_{2, k}=\left[\begin{array}{c}
0.5 \\
0.6+0.1 \cos (0.5 k)
\end{array}\right], B_{3, k}=\left[\begin{array}{c}
-0.6 \\
0.8
\end{array}\right] .
\end{aligned}
$$

It is easy to verify that

$$
\begin{aligned}
F_{1, k} & =\left[\begin{array}{cc}
0.2 & 0 \\
0 & 0.15
\end{array}\right], F_{2, k}=\left[\begin{array}{cc}
0.3 & 0 \\
0 & 0.2
\end{array}\right] \\
S_{i, k} & =\left[\begin{array}{cc}
0.3 & 0 \\
0 & 0.3
\end{array}\right] .
\end{aligned}
$$

The parameters in (5) and (11) are set as

$$
\begin{aligned}
C_{1, k} & =\left[\begin{array}{ll}
0.3 & 0.2
\end{array}\right], D_{1, k}=0.5, E_{1, k}=\left[\begin{array}{ll}
0.5 & 0.5
\end{array}\right] \\
C_{2, k} & =\left[\begin{array}{ll}
-0.2 & 0.1
\end{array}\right], D_{2, k}=0.5, E_{2, k}=\left[\begin{array}{ll}
0.3 & 0.4
\end{array}\right] \\
C_{3, k} & =\left[\begin{array}{ll}
0.1 & 0.1
\end{array}\right], D_{3, k}=0.6, E_{3, k}=\left[\begin{array}{ll}
0.4 & 0.6
\end{array}\right] .
\end{aligned}
$$

Let $\gamma=0.8, \alpha_{k}=0.5, \beta_{k}=0.4$ and $W=I_{15}$. For the inner coupling (3), $g_{1}$ and $g_{2}$ are, respectively, selected as $g_{1}=0.2$ and $g_{2}=0.3 . \psi_{1, k}, \psi_{2, k}$ and $\psi_{3, k}$ are mutually independent random sequences obeying uniform distribution over the interval $[0.1,0.9]$. As such, the means and variances of the random sequences $\psi_{i, k}$ are $\psi_{1}=\psi_{2}=\psi_{3}=0.4$ and $\sigma_{1}=\sigma_{2}=\sigma_{3}=0.0533$. According to Algorithm FHHE, the values of gain matrix $L_{i, k}(i=1,2,3 ; k=0,1,2, \ldots, 40)$ are recursively calculated and listed in Table I. It should be noted from Algorithm FHHE and Table I that the estimator gains at instant $k=40$ become zero because of the equality (30) and the preseted values $P_{41}=Q_{41}=0$.

The exogenous disturbance is $v(k)=2 \cos (k) e^{-0.02 k}$. The initial conditions of the measurements, system states and their estimations are given as $y_{1,0}=y_{2,0}=y_{3,0}=0$, $x_{1,0}=\left[\begin{array}{ll}0.1 & -0.1\end{array}\right]^{T}, x_{2,0}=\left[\begin{array}{ll}0.2 & 0.1\end{array}\right]^{T}, x_{3,0}=\left[\begin{array}{ll}0.1 & 0.2\end{array}\right]^{T}$, $\hat{x}_{1,0}=\left[\begin{array}{ll}-0.1 & 0.1\end{array}\right]^{T}, \hat{x}_{2,0}=\left[\begin{array}{ll}0.1 & -0.1\end{array}\right]^{T}$ and $\hat{x}_{3,0}=$ $\left[\begin{array}{ll}-0.1 & 0.1\end{array}\right]^{T}$. The responses of $x_{i, k}(I=1,2,3)$ and their estimates are illustrated in Figs. 1-3. The output estimation errors $\bar{z}_{i, k}(i=1,2,3)$ are shown in Fig. 4. It is evident

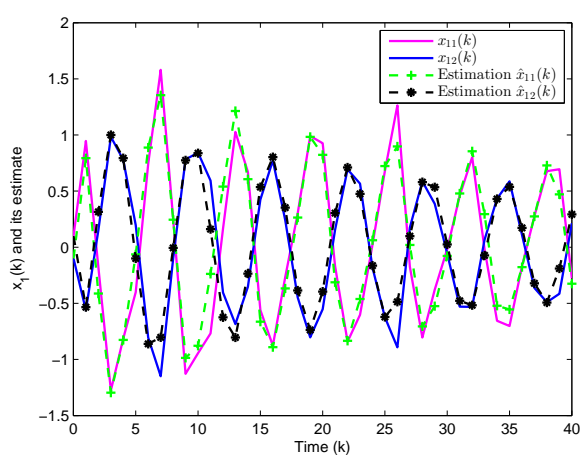

Fig. 1. $x_{1}(k)$ and its estimates

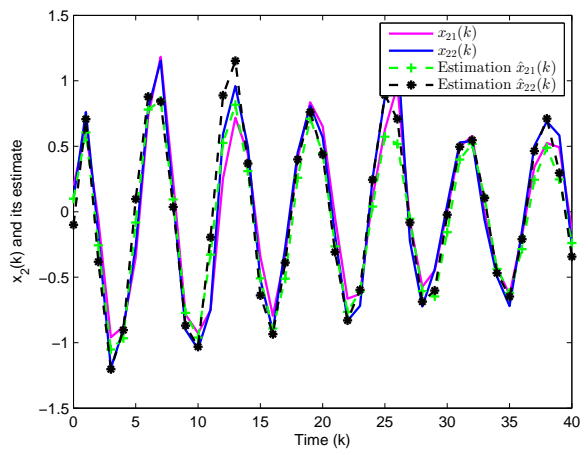

Fig. 2. $x_{2}(k)$ and its estimates

from Fig. 4 that the designed estimator achieves satisfactory performance over the prescribed finite horizon $[0,40]$.

\section{CONCLUSIONS}

In this paper, the finite-Horizon $H_{\infty}$ state estimation problem has been investigated for a class of stochastic coupled networks subject to random inner coupling variations and the Round-Robin scheduling protocol. In order to reduce the network communication burden, the Round-Robin transmission protocol has been applied to schedule the measurement signals from the sensors to the estimators. A novel linearization technique has been developed to deal with the system nonlinearities. Also, for the purpose of facilitating the theoretical

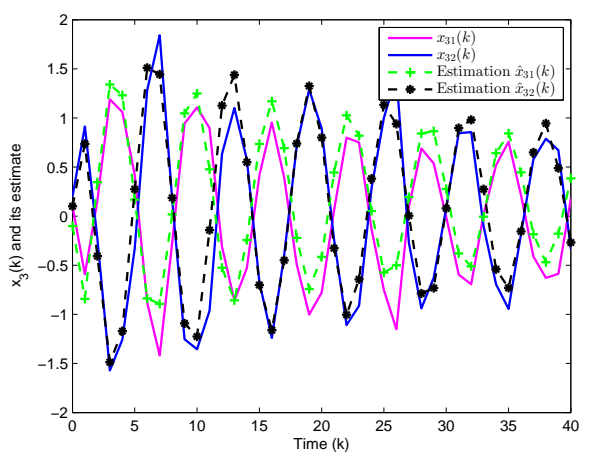

Fig. 3. $x_{3}(k)$ and its estimates 


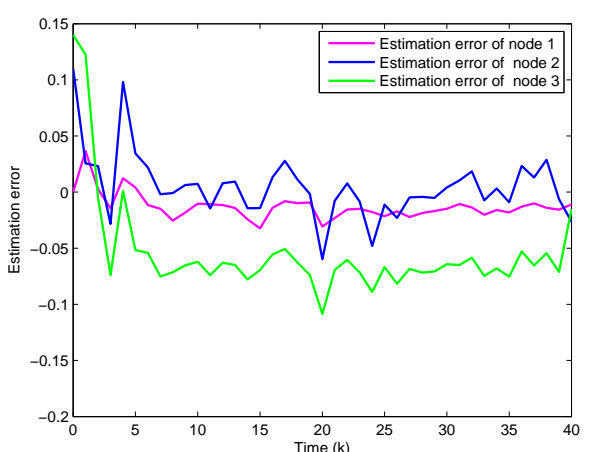

Fig. 4. The output estimation errors

analysis, some ingenious system transformation operations have been adopted such that the multiplicative noise term is included in the coefficient matrix rather than the augmented disturbance vector. By employing the backward Riccati difference equation technique, the desired finite-horizon $H_{\infty}$ state estimators have been determined by recursively solving a set of coupled difference equations. The effectiveness of the state estimation algorithm has been finally verified by a numerical example. Future research topics would be to extend the main results to more complicated systems with different performance specifications (e.g. variance-constrained performance, mixed $H_{2} / H_{\infty}$ index). Similarly, we can also investigate the finite-horizon $H_{\infty}$ state estimation problems for stochastic coupled networks based on event-triggered mechanism and other communication scheduling protocols [4], [6], [10], [24], [35], [46]-[50].

\section{APPENDIX}

\section{A. Proof of Theorem 1}

Proof: Define the following index

$$
\bar{J}_{k} \triangleq \mathbf{E}\left\{\eta_{k+1}^{T} P_{k+1} \eta_{k+1}-\eta_{k}^{T} P_{k} \eta_{k}\right\} .
$$

Along the dynamics of auxiliary system (17), the index (32) is calculated by

$$
\begin{aligned}
\bar{J}_{k}= & \mathbf{E}\left\{\eta _ { k } ^ { T } \left[\left(\mathcal{A}_{k}+\mathbf{F}_{k}\right)^{T} P_{k+1}\left(\mathcal{A}_{k}+\mathbf{F}_{k}\right)\right.\right. \\
& \left.+\Delta_{k}^{T} P_{k+1} \Delta_{k}+2\left(\mathcal{A}_{k}+\mathbf{F}_{k}\right)^{T} P_{k+1} \Delta_{k}-P_{k}\right] \eta_{k} \\
& +2 \eta_{k}^{T}\left(\mathcal{A}_{k}+\mathbf{F}_{k}+\Delta_{k}\right)^{T} P_{k+1} \mathbf{B}_{k} \mathbf{v}_{k} \\
& \left.+\mathbf{v}_{k}^{T} \mathbf{B}_{k}^{T} P_{k+1} \mathbf{B}_{k} \mathbf{v}_{k}\right\} .
\end{aligned}
$$

By virtue of $\mathbf{E}\left\{\Delta_{k}\right\}=0$ and denoting $\mathbb{A}_{k} \triangleq \mathcal{A}_{k}+\mathbf{F}_{k}$, (33) is simplified as

$$
\begin{aligned}
\bar{J}_{k}= & \mathbf{E}\left\{\eta_{k}^{T}\left[\mathbb{A}_{k}^{T} P_{k+1} \mathbb{A}_{k}+\Delta_{k}^{T} P_{k+1} \Delta_{k}-P_{k}\right] \eta_{k}\right. \\
& \left.+2 \eta_{k}^{T} \mathbb{A}_{k}^{T} P_{k+1} \mathbf{B}_{k} \mathbf{v}_{k}+\mathbf{v}_{k}^{T} \mathbf{B}_{k}^{T} P_{k+1} \mathbf{B}_{k} \mathbf{v}_{k}\right\}
\end{aligned}
$$

In addition, one further calculates

$$
\begin{aligned}
& \mathbf{E}\left\{\eta_{k}^{T} \Delta_{k}^{T} P_{k+1} \Delta_{k} \eta_{k}\right\} \\
= & \mathbf{E}\left\{\eta_{k}^{T} \operatorname{diag}\left\{\tilde{R}_{k}^{T}\left(P_{k+1}^{11}+2 P_{k+1}^{13}+P_{k+1}^{33}\right) \tilde{R}_{k}, 0,0\right\} \eta_{k}\right\}
\end{aligned}
$$

where $\tilde{R}_{k}=R \otimes\left(G_{k}-G\right)$ and $P_{k+1}^{\mu \nu}$ is the $(\mu, \nu)$-th entry of matrix $P_{k+1}(\mu, \nu=1,2,3)$.

Applying Lemma 1 and the mutual independence of $\psi_{q, k}(q=1,2, \cdots, n)$ in (3), one observes that

$$
\mathbf{E}\left\{\eta_{k}^{T} \Delta_{k}^{T} P_{k+1} \Delta_{k} \eta_{k}\right\}=\mathbf{E}\left\{\eta_{k}^{T} \overline{\mathbf{G}}^{T} P_{k+1} \overline{\mathbf{G}} \eta_{k}\right\} .
$$

It is obvious that $\mathbf{B}_{k}$ in (18) is decomposed into two parts as follows

$$
\begin{aligned}
\mathbf{B}_{k} & =\left[\begin{array}{lll}
\mathcal{B}_{k} & \alpha_{k}^{-1} I & 0
\end{array}\right]+\left[\begin{array}{lll}
0 & 0 & \beta_{k}^{-1} I
\end{array}\right] w_{k} \\
& =\Xi_{k}+\Gamma_{k} w_{k}
\end{aligned}
$$

where the first and second parts indicate in deterministic and stochastic settings, respectively.

It follows from the statistical characteristics of $w_{k}$ that

$$
\mathbf{E}\left\{2 \eta_{k}^{T} \mathbb{A}_{k}^{T} P_{k+1} \mathbf{B}_{k} \mathbf{v}_{k}\right\}=\mathbf{E}\left\{2 \eta_{k}^{T} \mathbb{A}_{k}^{T} P_{k+1} \Xi_{k} \mathbf{v}_{k}\right\}
$$

and

$$
\mathbf{E}\left\{\mathbf{v}_{k}^{T} \mathbf{B}_{k}^{T} P_{k+1} \mathbf{B}_{k} \mathbf{v}_{k}\right\}=\mathbf{E}\left\{\mathbf{v}_{k}^{T} X_{k} \mathbf{v}_{k}\right\}
$$

where $X_{k}$ is given in (20). Then, one derives

$$
\begin{aligned}
\bar{J}_{k}= & \mathbf{E}\left\{\eta_{k}^{T}\left[\mathbb{A}_{k}^{T} P_{k+1} \mathbb{A}_{k}+\overline{\mathbf{G}}^{T} P_{k+1} \overline{\mathbf{G}}-P_{k}\right] \eta_{k}\right. \\
& \left.+2 \eta_{k}^{T} \mathbb{A}_{k}^{T} P_{k+1} \Xi_{k} \mathbf{v}_{k}+\mathbf{v}_{k}^{T} X_{k} \mathbf{v}_{k}\right\} .
\end{aligned}
$$

By adding the following zero term

$$
\begin{aligned}
& \left\|\bar{z}_{k}\right\|^{2}-\gamma^{2}\left(\left\|\mathbf{v}_{k}\right\|^{2}-\left\|\alpha_{k} \mathbb{F}_{k} \eta_{k}\right\|^{2}-\left\|\beta_{k} \mathbf{H}_{k} \eta_{k}\right\|^{2}\right) \\
& -\left\|\bar{z}_{k}\right\|^{2}+\gamma^{2}\left(\left\|\mathbf{v}_{k}\right\|^{2}-\left\|\alpha_{k} \mathbb{F}_{k} \eta_{k}\right\|^{2}-\left\|\beta_{k} \mathbf{H}_{k} \eta_{k}\right\|^{2}\right)
\end{aligned}
$$

to $\bar{J}_{k}$ results in

$$
\begin{aligned}
\bar{J}_{k}= & \mathbf{E}\left\{\eta _ { k } ^ { T } \left[\mathbb{A}_{k}^{T} P_{k+1} \mathbb{A}_{k}+\overline{\mathbf{G}}^{T} P_{k+1} \overline{\mathbf{G}}-P_{k}\right.\right. \\
& \left.+\mathcal{E}_{k}^{T} \mathcal{E}_{k}+\gamma^{2} \alpha_{k}^{2} \mathbb{F}_{k}^{T} \mathbb{F}_{k}+\gamma^{2} \beta_{k}^{2} \mathbf{H}_{k}^{T} \mathbf{H}_{k}\right] \eta_{k} \\
& +2 \eta_{k}^{T} \mathbb{A}_{k}^{T} P_{k+1} \Xi_{k} \mathbf{v}_{k}-\mathbf{v}_{k}^{T}\left(\gamma^{2} I-X_{k}\right) \mathbf{v}_{k}-\left\|\bar{z}_{k}\right\|^{2} \\
& \left.+\gamma^{2}\left(\left\|\mathbf{v}_{k}\right\|^{2}-\left\|\alpha_{k} \mathbb{F}_{k} \eta_{k}\right\|^{2}-\left\|\beta_{k} \mathbf{H}_{k} \eta_{k}\right\|^{2}\right)\right\}
\end{aligned}
$$

Moreover, invoking the completing-the-squares technique leads to

$$
\begin{aligned}
& 2 \eta_{k}^{T} \mathbb{A}_{k}^{T} P_{k+1} \Xi_{k} \mathbf{v}_{k}-\mathbf{v}_{k}^{T}\left(\gamma^{2} I-X_{k}\right) \mathbf{v}_{k} \\
= & \left(\mathbf{v}_{k}^{*}\right)^{T} \Omega_{k} \mathbf{v}_{k}^{*}-\left(\mathbf{v}_{k}-\mathbf{v}_{k}^{*}\right)^{T} \Omega_{k}\left(\mathbf{v}_{k}-\mathbf{v}_{k}^{*}\right)
\end{aligned}
$$


This article has been accepted for publication in a future issue of this journal, but has not been fully edited. Content may change prior to final publication. Citation information: DOI10.1109/TCYB.2020.3004288, IEEE Transactions on Cybernetics

where $\mathbf{v}_{k}^{*}=\Omega_{k}^{-1} \Xi_{k}^{T} P_{k+1} \mathbb{A}_{k} \eta_{k}$ and $\Omega_{k}=\gamma^{2} I-X_{k}$. Subsequently, we arrive at

$$
\begin{aligned}
\bar{J}_{k}= & \mathbf{E}\left\{\eta_{k+1}^{T} P_{k+1} \eta_{k+1}-\eta_{k}^{T} P_{k} \eta_{k}\right\} \\
= & \mathbf{E}\left\{\eta _ { k } ^ { T } \left[\mathbb{A}_{k}^{T} P_{k+1} \mathbb{A}_{k}+\overline{\mathbf{G}}^{T} P_{k+1} \overline{\mathbf{G}}+\mathcal{E}_{k}^{T} \mathcal{E}_{k}+\gamma^{2} \alpha_{k}^{2} \mathbb{F}_{k}^{T} \mathbb{F}_{k}\right.\right. \\
& \left.+\gamma^{2} \beta_{k}^{2} \mathbf{H}_{k}^{T} \mathbf{H}_{k}+\mathbb{A}_{k}^{T} P_{k+1} \Xi_{k} \Omega_{k}^{-1} \Xi_{k}^{T} P_{k+1} \mathbb{A}_{k}-P_{k}\right] \eta_{k} \\
& -\left(\mathbf{v}_{k}-\mathbf{v}_{k}^{*}\right)^{T} \Omega_{k}\left(\mathbf{v}_{k}-\mathbf{v}_{k}^{*}\right)-\left\|\bar{z}_{k}\right\|^{2} \\
& \left.+\gamma^{2}\left(\left\|\mathbf{v}_{k}\right\|^{2}-\left\|\alpha_{k} \mathbb{F}_{k} \eta_{k}\right\|^{2}-\left\|\beta_{k} \mathbf{H}_{k} \eta_{k}\right\|^{2}\right)\right\}
\end{aligned}
$$

Summing both sides of (36) from 0 to $T$ and noticing (21), it is easy to see that

$$
\begin{aligned}
& \mathbf{E}\left\{\eta_{T+1}^{T} P_{T+1} \eta_{T+1}-\eta_{0}^{T} P_{0} \eta_{0}\right\} \\
= & \sum_{k=0}^{T} \mathbf{E}\left\{-\left(\mathbf{v}_{k}-\mathbf{v}_{k}^{*}\right)^{T} \Omega_{k}\left(\mathbf{v}_{k}-\mathbf{v}_{k}^{*}\right)-\left\|\bar{z}_{k}\right\|^{2}\right. \\
& \left.+\gamma^{2}\left(\left\|\mathbf{v}_{k}\right\|^{2}-\left\|\alpha_{k} \mathbb{F}_{k} \eta_{k}\right\|^{2}-\left\|\beta_{k} \mathbf{H}_{k} \eta_{k}\right\|^{2}\right)\right\} .
\end{aligned}
$$

On the basis of (20), we deduce that

$$
\begin{aligned}
J_{2}= & \sum_{k=0}^{T} \mathbf{E}\left\{\left\|\bar{z}_{k}\right\|^{2}-\gamma^{2}\left(\left\|\mathbf{v}_{k}\right\|^{2}-\left\|\alpha_{k} \mathbb{F}_{k} \eta_{k}\right\|^{2}\right.\right. \\
& \left.\left.-\left\|\beta_{k} \mathbf{H}_{k} \eta_{k}\right\|^{2}\right)-\gamma^{2} \eta_{0}^{T} W \eta_{0}\right\} \\
= & \mathbf{E}\left\{-\eta_{T+1}^{T} P_{T+1} \eta_{T+1}-\eta_{0}^{T}\left(\gamma^{2} W-P_{0}\right) \eta_{0}\right. \\
& \left.-\sum_{k=0}^{T}\left(\mathbf{v}_{k}-\mathbf{v}_{k}^{*}\right)^{T} \Omega_{k}\left(\mathbf{v}_{k}-\mathbf{v}_{k}^{*}\right)\right\} .
\end{aligned}
$$

With the help of $P_{T+1}=0, \Omega_{k}>0$ and $P_{0}<\gamma^{2} W$, it is obvious that $J_{2}<0$ holds and this completes the proof of this theorem.

\section{B. Proof of Theorem 2}

Proof: Introduce a performance index as

$$
\tilde{J}_{k}=\mathbf{E}\left\{\eta_{k+1}^{T} Q_{k+1} \eta_{k+1}-\eta_{k}^{T} Q_{k} \eta_{k}\right\} .
$$

Noticing (23), (24) and $\mathbf{E}\left\{\Delta_{k}\right\}=0$, the above index is computed as

$$
\begin{aligned}
\tilde{J}_{k}= & \mathbf{E}\left\{\eta _ { k } ^ { T } \left[\left(\overline{\mathbb{A}}_{k}+\overline{\mathbb{B}}_{k} \overline{\mathbb{A}}_{k}+\Delta_{k}\right)^{T} Q_{k+1}\left(\overline{\mathbb{A}}_{k}+\overline{\mathbb{B}}_{k} \overline{\mathbb{A}}_{k}+\Delta_{k}\right)\right.\right. \\
& \left.-Q_{k}\right] \eta_{k}+\phi_{k}^{T} \mathcal{I}^{T}\left(\overline{\mathbb{B}}_{k}+I\right)^{T} Q_{k+1}\left(\overline{\mathbb{B}}_{k}+I\right) \mathcal{I} \phi_{k} \\
& \left.+2 \eta_{k}^{T}\left(\overline{\mathbb{A}}_{k}+\overline{\mathbb{B}}_{k} \overline{\mathbb{A}}_{k}+\Delta_{k}\right)^{T} Q_{k+1}\left(\overline{\mathbb{B}}_{k}+I\right) \mathcal{I} \phi_{k}\right\} \\
= & \mathbf{E}\left\{\eta _ { k } ^ { T } \left[\left(\overline{\mathbb{A}}_{k}+\overline{\mathbb{B}}_{k} \overline{\mathbb{A}}_{k}\right)^{T} Q_{k+1}\left(\overline{\mathbb{A}}_{k}+\overline{\mathbb{B}}_{k} \overline{\mathbb{A}}_{k}\right)\right.\right. \\
& \left.+\Delta_{k}^{T} Q_{k+1} \Delta_{k}-Q_{k}\right] \eta_{k}+2 \eta_{k}^{T}\left(\overline{\mathbb{A}}_{k}+\overline{\mathbb{B}}_{k} \overline{\mathbb{A}}_{k}\right)^{T} Q_{k+1} \\
& \left.\left(\overline{\mathbb{B}}_{k}+I\right) \mathcal{I} \phi_{k}+\phi_{k}^{T} \mathcal{I}^{T}\left(\overline{\mathbb{B}}_{k}+I\right)^{T} Q_{k+1}\left(\overline{\mathbb{B}}_{k}+I\right) \mathcal{I} \phi_{k}\right\} .
\end{aligned}
$$

As in the proof of Theorem 1, it is easy to observe that

$$
\mathbf{E}\left\{\eta_{k}^{T} \Delta_{k}^{T} Q_{k+1} \Delta_{k} \eta_{k}\right\}=\mathbf{E}\left\{\eta_{k}^{T} \overline{\mathbf{G}}^{T} Q_{k+1} \overline{\mathbf{G}} \eta_{k}\right\}
$$

where $\overline{\mathbf{G}}$ is given in Theorem 1 .

It follows from Lemma 2 that $\mathbf{E}\left\{w_{k}\right\}=\mathbf{E}\left\{w_{k}^{3}\right\}=0$, $\mathbf{E}\left\{w_{k}^{2}\right\}=1, \mathbf{E}\left\{w_{k}^{4}\right\}=3$ and

$$
\begin{aligned}
& \mathbf{E}\left\{\eta_{k}^{T}\left[\left(\overline{\mathbb{A}}_{k}+\overline{\mathbb{B}}_{k} \overline{\mathbb{A}}_{k}\right)^{T} Q_{k+1}\left(\overline{\mathbb{A}}_{k}+\overline{\mathbb{B}}_{k} \overline{\mathbb{A}}_{k}\right)\right] \eta_{k}\right\} \\
= & \mathbf{E}\left\{\eta_{k}^{T} \overline{\mathbb{A}}_{k}^{T}\left[Q_{k+1}+2 Q_{k+1} \overline{\mathbb{B}}_{k}+\overline{\mathbb{B}}_{k}^{T} Q_{k+1} \overline{\mathbb{B}}_{k}\right] \overline{\mathbb{A}}_{k} \eta_{k}\right\} \\
= & \mathbf{E}\left\{\eta_{k}^{T} \overline{\mathbb{A}}_{k}^{T}\left[Q_{k+1}+2 Q_{k+1} Y_{k} P_{k+1}+\overline{\mathbb{B}}_{k}^{T} Q_{k+1} \overline{\mathbb{B}}_{k}\right] \overline{\mathbb{A}}_{k} \eta_{k}\right\}
\end{aligned}
$$

where $Y_{k}$ is given in (20), and the last term of the above equality is shown as

$$
\begin{aligned}
& \mathbf{E}\left\{\eta_{k}^{T} \overline{\mathbb{A}}_{k}^{T} \overline{\mathbb{B}}_{k}^{T} Q_{k+1} \overline{\mathbb{B}}_{k} \overline{\mathbb{A}}_{k} \eta_{k}\right\} \\
= & \mathbf{E}\left\{\eta _ { k } ^ { T } \overline { \mathbb { A } } _ { k } ^ { T } P _ { k + 1 } \left(\Xi_{k} \Omega_{k}^{-1} \Xi_{k}^{T} Q_{k+1} \Xi_{k} \Omega_{k}^{-1} \Xi_{k}^{T}\right.\right. \\
& +4 \Xi_{k} \Omega_{k}^{-1} \Gamma_{k}^{T} Q_{k+1} \Gamma_{k} \Omega_{k}^{-1} \Xi_{k}^{T} \\
& +3 \Gamma_{k} \Omega_{k}^{-1} \Gamma_{k}^{T} Q_{k+1} \Gamma_{k} \Omega_{k}^{-1} \Gamma_{k}^{T} \\
& \left.\left.+2 \Gamma_{k} \Omega_{k}^{-1} \Gamma_{k}^{T} Q_{k+1} \Xi_{k} \Omega_{k}^{-1} \Xi_{k}^{T}\right) P_{k+1} \overline{\mathbb{A}}_{k} \eta_{k}\right\} \\
\triangleq & \mathbf{E}\left\{\eta_{k}^{T} \overline{\mathbb{A}}_{k}^{T} \mathbf{M}_{k} \overline{\mathbb{A}}_{k} \eta_{k}\right\}
\end{aligned}
$$

where

$$
\begin{aligned}
\mathbf{M}_{k}= & P_{k+1}\left(\Xi_{k} \Omega_{k}^{-1} \Xi_{k}^{T} Q_{k+1} \Xi_{k} \Omega_{k}^{-1} \Xi_{k}^{T}\right. \\
& +4 \Xi_{k} \Omega_{k}^{-1} \Gamma_{k}^{T} Q_{k+1} \Gamma_{k} \Omega_{k}^{-1} \Xi_{k}^{T} \\
& +3 \Gamma_{k} \Omega_{k}^{-1} \Gamma_{k}^{T} Q_{k+1} \Gamma_{k} \Omega_{k}^{-1} \Gamma_{k}^{T} \\
& \left.+2 \Gamma_{k} \Omega_{k}^{-1} \Gamma_{k}^{T} Q_{k+1} \Xi_{k} \Omega_{k}^{-1} \Xi_{k}^{T}\right) P_{k+1} \\
= & P_{k+1}\left(Y_{k} Q_{k+1} Y_{k}+4 \Xi_{k} \Omega_{k}^{-1} \Gamma_{k}^{T} Q_{k+1} \Gamma_{k} \Omega_{k}^{-1} \Xi_{k}^{T}\right. \\
& \left.+2 \Gamma_{k} \Omega_{k}^{-1} \Gamma_{k}^{T} Q_{k+1} \Gamma_{k} \Omega_{k}^{-1} \Gamma_{k}^{T}\right) P_{k+1} .
\end{aligned}
$$

Then, we obtain

$$
\begin{aligned}
& \mathbf{E}\left\{\eta_{k}^{T}\left[\left(\overline{\mathbb{A}}_{k}+\overline{\mathbb{B}}_{k} \overline{\mathbb{A}}_{k}\right)^{T} Q_{k+1}\left(\overline{\mathbb{A}}_{k}+\overline{\mathbb{B}}_{k} \overline{\mathbb{A}}_{k}\right)\right] \eta_{k}\right\} \\
= & \mathbf{E}\left\{\eta_{k}^{T} \overline{\mathbb{A}}_{k}^{T}\left[Q_{k+1}+2 Q_{k+1} Y_{k} P_{k+1}+\mathbf{M}_{k}\right] \overline{\mathbb{A}}_{k} \eta_{k}\right\} \\
\triangleq & \mathbf{E}\left\{\eta_{k}^{T} \overline{\mathbb{A}}_{k}^{T} Z_{k} \overline{\mathbb{A}}_{k} \eta_{k}\right\} .
\end{aligned}
$$

Similarly, we have

$$
\begin{aligned}
& \mathbf{E}\left\{2 \eta_{k}^{T}\left(\overline{\mathbb{A}}_{k}+\overline{\mathbb{B}}_{k} \overline{\mathbb{A}}_{k}\right)^{T} Q_{k+1}\left(\overline{\mathbb{B}}_{k}+I\right) \mathcal{I} \phi_{k}\right\} \\
= & \mathbf{E}\left\{2 \eta_{k}^{T} \overline{\mathbb{A}}_{k}^{T} Q_{k+1}\left(\overline{\mathbb{B}}_{k}+I\right) \mathcal{I} \phi_{k}\right. \\
& \left.+2 \eta_{k}^{T} \overline{\mathbb{A}}_{k}^{T} \overline{\mathbb{B}}_{k}^{T} Q_{k+1}\left(\overline{\mathbb{B}}_{k}+I\right) \mathcal{I} \phi_{k}\right\} \\
= & \mathbf{E}\left\{2 \eta_{k}^{T} \overline{\mathbb{A}}_{k}^{T} Q_{k+1}\left(Y_{k} P_{k+1}+I\right) \mathcal{I} \phi_{k}\right. \\
& \left.+2 \eta_{k}^{T} \overline{\mathbb{A}}_{k}^{T}\left(\mathbf{M}_{k}+P_{k+1} Y_{k} Q_{k+1}\right) \mathcal{I} \phi_{k}\right\}
\end{aligned}
$$


and

$$
\begin{aligned}
& \mathbf{E}\left\{\phi_{k}^{T} \mathcal{I}^{T}\left(\overline{\mathbb{B}}_{k}+I\right)^{T} Q_{k+1}\left(\overline{\mathbb{B}}_{k}+I\right) \mathcal{I} \phi_{k}\right\} \\
= & \mathbf{E}\left\{\phi_{k}^{T} \mathcal{I}^{T}\left[Q_{k+1}+2 Q_{k+1} Y_{k} P_{k+1}+\mathbf{M}_{k}\right] \mathcal{I} \phi_{k}\right\} \\
\triangleq & \mathbf{E}\left\{\phi_{k}^{T} \mathcal{I}^{T} Z_{k} \mathcal{I} \phi_{k}\right\} .
\end{aligned}
$$

Consequently, we have

$$
\begin{aligned}
\tilde{J}_{k}= & \mathbf{E}\left\{\eta _ { k } ^ { T } \left[\overline{\mathbb{A}}_{k}^{T} Z_{k} \overline{\mathbb{A}}_{k}+\overline{\mathbf{G}}^{T} Q_{k+1} \overline{\mathbf{G}}-Q_{k}+\mathcal{E}_{k}^{T} \mathcal{E}_{k}\right.\right. \\
& +\overline{\mathbb{A}}_{k}^{T}\left(\mathbf{M}_{k}+Q_{k+1} Y_{k} P_{k+1}\right) \mathcal{I} L_{k} \mathbf{C}_{k} \\
& \left.+\mathbf{C}_{k}^{T} L_{k}^{T} \mathcal{I}^{T}\left(\mathbf{M}_{k}+P_{k+1} Y_{k} Q_{k+1}\right) \overline{\mathbb{A}}_{k}\right] \eta_{k} \\
& +2 \eta_{k}^{T} \overline{\mathbb{A}}_{k}^{T} Q_{k+1}\left(Y_{k} P_{k+1}+I\right) \mathcal{I} \phi_{k} \\
& \left.+\phi_{k}^{T}\left(\mathcal{I}^{T} Z_{k} \mathcal{I}+I\right) \phi_{k}-\left\|\bar{z}_{k}\right\|^{2}-\left\|\phi_{k}\right\|^{2}\right\} .
\end{aligned}
$$

Denoting

$$
\begin{aligned}
\Psi_{k} & =\mathcal{I}^{T} Z_{k} \mathcal{I}+I \\
\phi_{k}^{*} & =\Psi_{k}^{-1} \mathcal{I}^{T} Q_{k+1}\left(Y_{k} P_{k+1}+I\right) \overline{\mathbb{A}}_{k} \eta_{k}
\end{aligned}
$$

we have

$$
\begin{aligned}
& 2 \eta_{k}^{T} \overline{\mathbb{A}}_{k}^{T} Q_{k+1}\left(Y_{k} P_{k+1}+I\right) \mathcal{I} \phi_{k}+\phi_{k}^{T}\left(\mathcal{I}^{T} Z_{k} \mathcal{I}+I\right) \phi_{k} \\
= & \left(\phi_{k}+\phi_{k}^{*}\right)^{T} \Psi_{k}\left(\phi_{k}+\phi_{k}^{*}\right)-\left(\phi_{k}^{*}\right)^{T} \Psi_{k} \phi_{k}^{*} .
\end{aligned}
$$

Thus, $\tilde{J}_{k}$ becomes as

$$
\begin{aligned}
\tilde{J}_{k}= & \mathbf{E}\left\{\eta _ { k } ^ { T } \left[\overline{\mathbb{A}}_{k}^{T} Z_{k} \overline{\mathbb{A}}_{k}+\overline{\mathbf{G}}^{T} Q_{k+1} \overline{\mathbf{G}}+\mathcal{E}_{k}^{T} \mathcal{E}_{k}-Q_{k}\right.\right. \\
& +\overline{\mathbb{A}}_{k}^{T}\left(\mathbf{M}_{k}+Q_{k+1} Y_{k} P_{k+1}\right) \mathcal{I} L_{k} \mathbf{C}_{k} \\
& +\mathbf{C}_{k}^{T} L_{k}^{T} \mathcal{I}^{T}\left(\mathbf{M}_{k}+P_{k+1} Y_{k} Q_{k+1}\right) \overline{\mathbb{A}}_{k} \\
& -\overline{\mathbb{A}}_{k}^{T}\left(P_{k+1} Y_{k}+I\right)^{T} Q_{k+1} \mathcal{I} \\
& \left.\times \Psi_{k}^{-1} \mathcal{I}^{T} Q_{k+1}\left(Y_{k} P_{k+1}+I\right) \overline{\mathbb{A}}_{k}\right] \eta_{k} \\
& \left.+\left(\phi_{k}+\phi_{k}^{*}\right)^{T} \Psi_{k}\left(\phi_{k}+\phi_{k}^{*}\right)-\left\|\bar{z}_{k}\right\|^{2}-\left\|\phi_{k}\right\|^{2}\right\} .
\end{aligned}
$$

According to (26) and (48), it follows readily that

$$
\begin{aligned}
& \mathbf{E}\left\{\eta_{T+1}^{T} Q_{T+1} \eta_{T+1}-\eta_{0}^{T} Q_{0} \eta_{0}\right\} \\
= & \sum_{k=0}^{T} \mathbf{E}\left\{\left(\phi_{k}+\phi_{k}^{*}\right)^{T} \Psi_{k}\left(\phi_{k}+\phi_{k}^{*}\right)\left\|\bar{z}_{k}\right\|^{2}-\left\|\phi_{k}\right\|^{2}\right\} .
\end{aligned}
$$

Combining (25), (39) and $Q_{T+1}=0$ leads to

$$
\tilde{J}=\sum_{k=0}^{T} \mathbf{E}\left\{\left(\phi_{k}+\phi_{k}^{*}\right)^{T} \Psi_{k}\left(\phi_{k}+\phi_{k}^{*}\right)\right\}+\eta_{0}^{T} Q_{0} \eta_{0}
$$

and we see immediately that $\phi_{k}=-\phi_{k}^{*}$, or

$$
L_{k} \mathbf{C}_{k}=-\Psi_{k}^{-1} \mathcal{I}^{T} Q_{k+1}\left(Y_{k} P_{k+1}+I\right) \overline{\mathbb{A}}_{k}
$$

minimizes the performance index $\tilde{J}$ as $\eta_{0}^{T} Q_{0} \eta_{0}$. Meanwhile, from (38) with $P_{T+1}=0$, the cost of $J_{2}$ is determined by $\eta_{0}^{T}\left(P_{0}-\gamma^{2} W\right) \eta_{0}$. The proof of this theorem is thus fulfilled.

\section{REFERENCES}

[1] A. O. Alweimine, O. Bamaarouf, A. Rachadi, and H. Ez-Zahraouy, Local routing protocols performance for computer virus elimination in complex networks, Physica A: Statistical Mechanics and its Applications, vol. 536, art. no. 120984, Dec. 2019.

[2] Y. Bar-Shalom, X. R. Li, and T. Kirubarajan, Estimation with Applications to Tracking and Navigation, John Wiley \& Sons, Inc., 2001.

[3] D. A. Burbano-L, G. Russo and M. di Bernardo, Pinning controllability of complex network systems with noise, IEEE Transactions on Neural Networks and Learning Systems, vol. 6, no. 2, pp. 874-883, Jun. 2019.

[4] B. Chen, G. Hu, D. W. C. Ho, and L. Yu, Distributed Kalman filtering for time-varying discrete sequential systems, Automatica, vol. 99, pp. 228236, Jan. 2019.

[5] Y. Chen, Z. Wang, W. Qian, and F. E. Alsaadi, Finite-horizon $H_{\infty}$ filtering for switched time-varying stochastic systems with random sensor nonlinearities and packet droputs, Signal Processing, vol. 138, pp. 138-145, 2017.

[6] Y. Chen, Z. Wang, and L. Wang, Mixed $H_{2} / H_{\infty}$ state estimation for discrete-time switched complex networks with random coupling strengths through redundant channels, IEEE Transactions on Neural Networks and Learning Systems, online, DOI: 10.1109/TNNLS.2019.2952249

[7] Y. Chen, Z. Wang, Y. Yuan, and P. Date, Distributed $H_{\infty}$ filtering for switched stochastic delayed systems over sensor networks with fading measurements, IEEE Transactions on Cybernetics, vol. 50, no. 1, pp. $2-$ 14, Jan. 2020.

[8] D. Ding, Z. Wang, H. Dong, and H. Shu, Distributed $H_{\infty}$ state estimation with stochastic parameters and nonlinearities through sensor networks: The finite-horizon case, Automatica, vol. 48, no. 8, pp. 15751585, Aug. 2012.

[9] D. Ding, Z. Wang, J. Lam, and B. Shen, Finite-horizon $H_{\infty}$ control for discrete systems with randomly cccurring nonlinearities and fading measurements, IEEE Transactions on Automatic Control, vol. 60, no. 9, pp. 2488-2493, Sep. 2015.

[10] H. Dong, N. Hou, Z. Wang, and W. Ren, Variance-constrained state estimation for complex networks with randomly varying topologies, IEEE Transactions on Neural Networks and Learning Systems, vol. 29, no. 7, pp. 2757-2768, Jul. 2018.

[11] X. Ge, Q.-L. Han, X.-M. Zhang, L. Ding, and F. Yang, Distributed eventtriggered estimation over sensor networks: A survey, IEEE Transactions on Cybernetics, vol. 50, no. 3, pp. 1306-1320, Mar. 2020.

[12] J. M. Hofman, A. Sharma, and D. J. Watts, Prediction and explanation in social systems, Science, vol. 355, no. 6324, pp. 486-488, 2017.

[13] R. A. Horn and C. R. Johnson, Topic in Matrix Analysis, New York: Cambridge University Press, 1991.

[14] N. Hou, Z. Wang, D. W. C. Ho and H. Dong, Robust partial-nodes-based state estimation for complex networks under deception attacks, IEEE Transactions on Cybernetics, in press, DOI:10.1109/TCYB.2019.2918760.

[15] W. Li, Y. Jia, and J. Du, Recursive state estimation for complex networks with random coupling strength, Neurocomputing, vol. 219, pp. 1-8, 2017.

[16] W. Li, Y. Jia, and J. Du, Variance-constrained state estimation for nonlinearly coupled complex networks, IEEE Transactions on Cybernetics, vol. 48, no. 2, pp. 818-824, Feb. 2018.

[17] S. Liu, Z. Wang, Y. Chen, and G. Wei, Protocol-based unscented Kalman filtering in the presence of stochastic uncertainties, IEEE Transactions on Automatic Control, vol. 65, no. 3, pp. 1303-1309, Mar. 2020.

[18] X. Liu, D. W. C. Ho, Q. Song, and W. Xu, Finite/fixed-time pinning synchronization of complex networks with stochastic disturbances, IEEE Transactions on Cybernetics, vol. 49, no. 6, pp. 2398-2403, Jun. 2019.

[19] X. Lu and B. K. Szymanski, A Regularized Stochastic Block Model for the robust community detection in complex networks, Scientific Reports, vol. 9, Sep. 2019, Art. no. 13247.

[20] F. Radicchi and C. Castellano, Uncertainty reduction for stochastic processes on complex networks, Physical Review Letters, vol. 120, no. 19, May 2018, Art. no. 198301.

[21] B. Shen, S. X. Ding, and Z. Wang, Finite-horizon $H_{\infty}$ fault estimation for uncertain linear discrete time-varying systems with known inputs, IEEE Transactions on Circuits and Systems-II: Express Briefs, vol. 60, no. 12 , pp. 902-906, Dec. 2013.

[22] B. Shen, Z. Wang, D. Ding, and H. Shu, $H_{\infty}$ state estimation for complex networks with uncertain inner coupling and incomplete measurements, IEEE Transactions on Neural Networks and Learning Systems, vol. 24, no. 12, pp. 2027-2037, Dec. 2013. 
[23] B. Shen, Z. Wang, D. Wang and H. Liu, Distributed state-saturated recursive filtering over sensor networks under Round-Robin protocol, IEEE Transactions on Cybernetics, in press, DOI: 10.1109/TCYB.2019.2932460.

[24] Y. Shen, Z. Wang, B. Shen, F. E. Alsaadi and F. E. Alsaadi, Fusion estimation for multi-rate linear repetitive processes under weighted Try-Once-Discard protocol, Information Fusion, vol. 55, pp. 281-291, Mar. 2020.

[25] L. Sheng, Y. Niu, and M. Gao, Distributed resilient filtering for timevarying systems over sensor networks subject to Round-Robin/stochastic protocol, ISA Transactions, vol. 87, pp. 55-67, Apr. 2019.

[26] L. Sheng, Y. Niu, L. Zou, Y. Liu, and F. E. Alsaadi, Finite-horizon state estimation for time-varying complex networks with random coupling strengths under Round-Robin protocol, Journal of The Franklin Institute, vol. 355, no. 15, pp. 7417-7442, Oct. 2018.

[27] S. Trimpe, Event-based state estimation: an emulation-based approach, IET Control Theory \& Applications, vol. 11, no. 11, pp. 1684-1693, 2017.

[28] X. Wan, Z. Wang, M. Wu, and X. Liu, $H_{\infty}$ state estimation for discretetime nonlinear singularly perturbed complex networks under the RoundRobin protocol, IEEE Transactions on Neural Networks and Learning Systems, vol. 30, no. 2, pp. 415-426, Feb. 2019.

[29] F. Wang and J. Liang, Constrained $H_{\infty}$ estimation for time-varying networks with hybrid incomplete information, International Journal of Robust and Nonlinear Control, vol. 28, no. 2, pp. 699-715, 2018.

[30] J.-L. Wang, Z. Qin, H.-N. Wu, and T. Huang, Finite-time synchronization and $H_{\infty}$ synchronization of multiweighted complex networks with adaptive state couplings, IEEE Transactions on Cybernetics, vol. 50, no. 2, pp. 600-612, Feb. 2020.

[31] L. Wang, Z. Wang, Q.-L. Han, and G. Wei, Synchronization control for a class of discrete-time dynamical networks with packet dropouts: A coding-decoding-based approach, IEEE Transactions on Cybernetics, vol. 48, no. 8, pp. 2437-2448, Aug. 2018.

[32] M. Wang, Z. Wang, Y. Chen and W. Sheng, Event-based adaptive neural tracking control for discrete-time stochastic nonlinear systems: A triggering threshold compensation strategy, IEEE Transactions on Neural Networks and Learning Systems, vol. 31, no. 6, pp. 1968-1981, Jun. 2020.

[33] S. Wang, H. Fang, and X. Tian, Event-based robust state estimator for linear time-varying system with uncertain observations and randomly occurring uncertainties, Journal of the Franklin Institute, vol. 354, no. 3, pp. 1403-1420, 2017.

[34] D. J. Watts and S. H. Strogatz, Collective dynamics of "small-world" networks, Nature, vol. 393, no. 6684, pp. 440-442, 1998.

[35] G. Wei, S. Liu, L. Wang, and Y. Wang, Event-based distributed setmembership filtering for a class of time-varying non-linear systems over sensor networks with saturation effects, International Journal of General Systems, vol. 45, no. 5, pp. 532-547, 2016.

[36] G. Wen, Y. Wan, J. Cao, T. Huang, and W. Yu, Master-slave synchronization of heterogeneous systems under scheduling communication, IEEE Transactions on Systems, Man, and Cybernetics: Systems, vol. 48, no. 3, pp. 473-484, Mar. 2018.

[37] Z.-G. Wu, P. Shi, H. Su, and J. Chu, Delay-dependent stability analysis for switched neural networks with time-varying delay, IEEE Transactions on Systems, Man, and Cybernetics, Part B: Cybernetics, vol. 41, no. 6, pp. 1522-1530, Dec. 2011.

[38] Y. Xu, R. Lu, P. Shi, H. Li, and S. Xie, Finite-time distributed state estimation over sensor networks with Round-Robin protocol and fading channels, IEEE Transactions on Cybernetics, vol. 48, no. 1, pp. 336345, Jan. 2018.

[39] Y. Xu, R. Lu, H. Peng, K. Xie, and A. Xue, Asynchronous dissipative state estimation for stochastic complex networks with quantized jumping coupling and uncertain measurements, IEEE Transactions on Neural Networks and Learning Systems, vol. 28, no. 2, pp. 268-277, Feb. 2017.

[40] Y. Xu, R. Lu, P. Shi, J. Tao, and S. Xie, Robust estimation for neural networks with randomly occurring distributed delays and Markovian jump coupling, IEEE Transactions on Neural Networks and Learning Systems, vol. 29, no. 4, pp. 845-855, Apr. 2018.

[41] Y. Xu, H. Su, Y.-J. Pan, Z.-G. Wu, and W. Xu, Stability analysis of networked control systems with round-robin scheduling and packet dropouts, Journal of the Franklin Institute, vol. 350, pp. 2013-2027, 2013.

[42] H. Zhang, J. Hu, H. Liu, X. Yu, and F. Liu, Recursive state estimation for time-varying complex networks subject to missing measurements and stochastic inner coupling under random access protocol, Neurocomputing, vol. 346, pp. 48-57, Jun. 2019.
[43] P. Zhang, Y. Yuan, H. Yang, and H. Liu, Near-Nash equilibrium control strategy for discrete-time nonlinear systems with Round-Robin protocol, IEEE Transactions on Neural Networks and Learning Systems, vol. 30, no. 8, pp. 2478-2492, Aug. 2019.

[44] X.-M. Zhang, Q.-L. Han, X. Ge, D. Ding, L. Ding, D. Yue, and C. Peng, Networked control systems: a survey of trends and techniques, IEEE/CAA Journal of Automatica Sinica, vol. 7, no. 1, pp. 1-17, 2020

[45] W. Zhang, X. Yang, and C. Li, Fixed-time stochastic synchronization of complex networks via continuous control, IEEE Transactions on Cybernetics, vol. 49, no. 8, pp. 3099-3104, Aug. 2019.

[46] D. Zhao, Z. Wang, Y. Chen and G. Wei, Proportional-integral observer design for multi-delayed sensor-saturated recurrent neural networks: A dynamic event-triggered protocol, IEEE Transactions on Cybernetics, in press, 10.1109/TCYB.2020.2969377.

[47] D. Zhao, Z. Wang, D. W. C. Ho and G. Wei, Observer-based PID security control for discrete time-delay systems under cyber-attacks, IEEE Transactions on Systems, Man, and Cybernetics-Systems, in press, DOI: 10.1109/TSMC.2019.2952539.

[48] L. Zou, Z. Wang, Q.-L. Han, and D.H. Zhou, Recursive filtering for time-varying systems with random access protocol, IEEE Transactions on Automatic Control, vol. 64, no. 2, pp. 720-727, Feb. 2019.

[49] L. Zou, Z. Wang, J. Hu and D. H. Zhou, Moving horizon estimation with unknown inputs under dynamic quantization effects, IEEE Transactions on Automatic Control, in press, DOI: 10.1109/TAC.2020.2968975.

[50] L. Zou, Z. Wang, Q.-L. Han and D. Zhou, Moving horizon estimation for networked time-delay systems under Round-Robin protocol, IEEE Transactions on Automatic Control, vol. 64, no. 12, pp. 5191-5198, Dec. 2019.

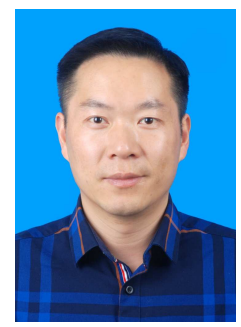

Yun Chen received the B.E. degree in thermal engineering in 1999 from Central South University of Technology (Central South University), Changsha, China, and the M.E. degree in engineering thermal physics in 2002 and Ph.D. degree in control science and engineering in 2008, both from Zhejiang University, Hangzhou, China.

From August 2009 to August 2010, he was a visiting fellow with the School of Computing, Engineering and Mathematics, University of Western Sydney, Australia. From December 2016 to December 2017, he was an academic visitor with the Department of Mathematics, Brunel University London, UK. In 2002, he joined Hangzhou Dianzi University, China, where he is currently a Professor. His research interests include stochastic and hybrid systems, robust control and filtering.

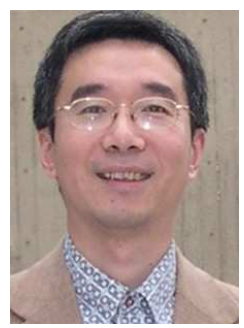

Zidong Wang (SM'03-F'14) was born in Jiangsu, China, in 1966. He received the B.Sc. degree in mathematics in 1986 from Suzhou University, Suzhou, China, and the M.Sc. degree in applied mathematics in 1990 and the Ph.D. degree in electrical engineering in 1994, both from Nanjing University of Science and Technology, Nanjing, China.

$\mathrm{He}$ is currently a Professor of Dynamical Systems and Computing in the Department of Computer Science, Brunel University London, U.K. From 1990 to 2002, he held teaching and research appointments in universities in China, Germany and the UK. Prof. Wang's research interests include dynamical systems, signal processing, bioinformatics, control theory and applications. He has published around 600 papers in refereed international journals. He is a holder of the Alexander von Humboldt Research Fellowship of Germany, the JSPS Research Fellowship of Japan, William Mong Visiting Research Fellowship of Hong Kong.

Prof. Wang serves (or has served) as the Editor-in-Chief for Neurocomputing, Deputy Editor-in-Chief for International Journal of Systems Science, and an Associate Editor for 12 international journals including IEEE Transactions on Automatic Control, IEEE Transactions on Control Systems Technology, IEEE Transactions on Neural Networks, IEEE Transactions on Signal Processing, and IEEE Transactions on Systems, Man, and Cybernetics-Part C. He is a Fellow of the IEEE, a Fellow of the Royal Statistical Society and a member of program committee for many international conferences. 


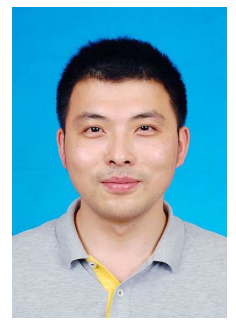

Licheng Wang received the B.Sc. degree in automation in 2011 from Weifang University, Weifang, China, the M.Sc. degree and the Ph.D. degree in control science and control engineering from University of Shanghai for Science and Technology, Shanghai, China, in 2014 and 2019, respectively.

From Nov. 2016 to Nov. 2018, he was a visiting Ph.D. student in the Department of Electronic and Computer Engineering at Brunel University London in the UK. He is currently a Post-Doctoral Research Fellow with the Department of Control Science and Engineering, University of Shanghai for Science and Technology, Shanghai, China. His research interests include nonlinear stochastic control and filtering, as well as complex networks and sensor networks.

Dr. Wang is currently a reviewer for some international journals.

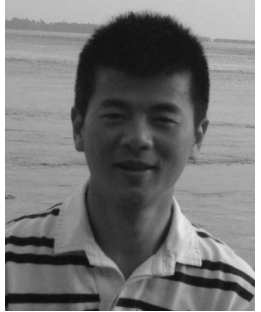

and machine learning.

Weiguo Sheng rreceived the M.Sc. degree in information technology from the University of Nottingham, Nottingham, U.K., in 2002, and the Ph.D. degree in computer science from Brunel University London, Uxbridge, U.K., in 2005.

He was a Researcher with the University of Kent, Canterbury, U.K., and Royal Holloway, University of London, Egham, U.K. He is currently a Professor with Hangzhou Normal University, Hangzhou, China. His research interests include evolutionary computation, data mining/clustering, pattern recognition, 